\title{
Micro-invasive glaucoma surgery (MIGS): a review of surgical procedures using stents
}

\author{
This article was published in the following Dove Press journal: \\ Clinical Ophthalmology \\ 29 August 2017 \\ Number of times this article has been viewed
}

\author{
Lutz E Pillunat' \\ Carl Erb² \\ Anselm GM Jünemann ${ }^{3}$ \\ Friedemann Kimmich ${ }^{4}$ \\ 'Department of Ophthalmology, \\ University Hospital Carl Gustav \\ Carus, Dresden, ${ }^{2}$ Augenklinik am \\ Wittenbergplatz, Berlin, Germany; \\ ${ }^{3}$ Department of Ophthalmology \\ University of Rostock, Rostock, \\ Germany; ${ }^{4}$ eyecons, Karlsruhe, \\ Germany
}

Correspondence: Lutz E Pillunat Department of Ophthalmology, University Hospital Carl Gustav Carus Fetscherstrasse 74, 01307 Dresden, Germany

Tel +4935 I 458338 I

Fax +49 35I 4584335

Email augendirektion@uniklinikumdresden.de

\begin{abstract}
Over the last decade several novel surgical treatment options and devices for glaucoma have been developed. All these developments aim to cause as little trauma as possible to the eye, to safely, effectively, and sustainably reduce intraocular pressure (IOP), to produce reproducible results, and to be easy to adopt. The term "micro-invasive glaucoma surgery (MIGS)" was used for summarizing all these procedures. Currently MIGS is gaining more and more interest and popularity. The possible reduction of the number of glaucoma medications, the ab interno approach without damaging the conjunctival tissue, and the probably safer procedures compared to incisional surgical methods may explain the increased interest in MIGS. The use of glaucoma drainage implants for lowering IOP in difficult-to-treat patients has been established for a long time, however, a variety of new glaucoma micro-stents are being manufactured by using various materials and are available to increase aqueous outflow via different pathways. This review summarizes published results of randomized clinical studies and extensive case report series on these devices, including Schlemm's canal stents (iStent ${ }^{\circledR}$, iStent $^{\circledR}$ inject, Hydrus), suprachoroidal stents (CyPass ${ }^{\circledR}$, iStent ${ }^{\circledR}$ Supra), and subconjunctival stents (XEN). The article summarizes the findings of published material on efficacy and safety for each of these approaches.
\end{abstract}

Keywords: glaucoma, micro-invasive glaucoma surgery, MIGS, iStent, iStent inject, CyPass, Hydrus, XEN

\section{Introduction}

Glaucoma is one of the leading causes of blindness worldwide. ${ }^{1}$ Several studies show that elevated intraocular pressure (IOP) is an important risk factor for glaucoma and the progression of the disease. ${ }^{2,3}$ It could also be demonstrated that lowering of elevated IOP can reduce the risk for progression. ${ }^{4,5}$ Today it is well accepted to initiate the treatment of patients with glaucoma and ocular hypertension with a medical therapy. If target pressure levels are not achieved and/or the disease is progressing despite using drug combinations, the next step on the therapeutic stepladder is to lower the IOP surgically. ${ }^{6}$ Despite having a proven record of efficacy and safety, the use of glaucoma medications may cause systemic and local side effects such as ocular surface disease and ocular allergy. ${ }^{7,8}$ Approximately 50\% of glaucoma patients require multiple medical treatments with different drugs. ${ }^{9}$ Local and systemic side effects, the use of different medications, and the overall complexity of the treatment-scheme may negatively impact adherence and persistance. ${ }^{10-13}$ Over the last several years new devices for micro-invasive glaucoma surgery (MIGS) were developed and have gained more and more interest. According to Saheb and Ahmed, the term MIGS refers to a group of surgical procedures which share five preferable qualities: an ab interno approach through a clear corneal incision which 
spares the conjunctiva of incision, a minimally traumatic procedure to the target tissue, an IOP lowering efficacy that justifies the approach, a high safety profile avoiding serious complications compared to other glaucoma surgeries, and a rapid recovery with minimal impact on the patient's quality of life. ${ }^{14}$ However, there is currently no single common and widely accepted definition of MIGS. In a workshop of the American Glaucoma Society and the US Food and Drug Administration (FDA) held in February 2014, the term "minimally invasive glaucoma surgery" was characterized by the implantation of a surgical device intended to lower IOP via an outflow mechanism with either an ab interno or ab externo approach, associated with very little or no scleral dissection. In this approach, to define MIGS procedures involving a significant scleral dissection was not classified under the term MIGS. ${ }^{15}$ All devices do not require a scleral incision and are placed ab interno by using a clear corneal incision. Thus they are frequently used in combination with phacoemulsification and intraocular lens implantation (PE/IOL). MIGS is intended to achieve lower IOP in patients with glaucoma with shorter surgical time, and ideally to achieve a medication sparing effect. All devices work by increasing the outflow of aqueous humor from the anterior chamber, either by directly accessing Schlemm's canal ${ }^{16-18}$ or shunting aqueous humor to the suprachorida ${ }^{19}$ or subconjunctival space. ${ }^{20}$ Complications such as hypotony, hyphema, infections of the bleb, revisions of the bleb, and endophthalmitis may occur in up to $35 \%$ of patients treated with conventional glaucoma surgery, ie, trabeculectomy (TE) ${ }^{21}$ MIGS may avoid those complications and therefore provide a valuable treatment option in glaucoma patients, however, the incidence and types of adverse events and possible complications may differ between the different types of MIGS procedures. The technical characteristics of different micro-implants which are available and under development are summarized in Table 1.

\section{Procedures targeting the trabecular outflow}

Currently, three devices (iStent, iStent inject [Glaukos Inc., Laguna Hills, CA, USA], and Hydrus [Ivantis Inc., Irvine, CA, USA]) target the juxtacanalicular part of the trabecular meshwork, which is believed to represent the greatest resistance to aqueous humor outflow in patients with open-angle glaucoma (OAG). ${ }^{22,23}$ These micro-invasive procedures allow for more direct access to aqueous humor from the anterior chamber into Schlemm's canal. One limitation of all of these procedures is that the postoperative IOP cannot fall below the episcleral venous pressure (EVP), which is difficult to evaluate but is reported in different studies in a range of 7.6 to $9.1 \mathrm{mmHg} .{ }^{24-26}$ It was also demonstrated that EVP may be elevated in some glaucoma patients. ${ }^{27}$

\section{iStent ${ }^{\circledR}$ and iStent $^{\circledR}$ inject}

iStent is the first generation trabecular bypass device that is manufactured by Glaukos Inc. This device connects the anterior chamber with Schlemm's canal. The iStent has CE-mark and was approved in 2012 by the FDA. In Europe, iStent is approved as a stand-alone device or for use in combined cataract/MIGS procedures. The product has a size of $1 \times 0.3 \mathrm{~mm}$, is made from heparin-coated, non-magnetic titanium, and is provided pre-loaded in an inserter (Table 1).

\section{Surgical procedure with iStent}

The iStent is delivered in an inserter which consists of a 26-gauge disposable instrument which contains the iStent on the tip. The stent is often implanted in a combined procedure with a cataract surgery. The device is implanted through the same temporal clear corneal incision used for PE/IOL. The leading edge of iStent is inserted through the trabecular meshwork into Schlemm's canal at the nasal position where the tip of the stent is pointing inferiorly. By pushing a button on the inserter, the device is released. There is a right- and left-eye model which are distinguished by the direction of the foot (Figure 1). Usually, postoperative anti-inflammatory and anti-infective topical medications are applied for approximately 4 weeks.

\section{Surgical procedure with iStent inject}

iStent inject is a much smaller second generation model (Figure 1). With a length of only $360 \mu \mathrm{m}$ and a diameter of $230 \mu \mathrm{m}$, a single iStent inject stent is currently the smallest medical implant approved for use in the human body during surgical procedures. The iStent inject stents are delivered in an injector system which injects the stents automatically into Schlemm's canal through a stainless steel insertion tube. The injector is released by the surgeon by pressing a button. The G2-M-IS injector system contains two stents, allowing the insertion of both stents from one injector during the same surgical procedure. Usually two iStent inject stents are implanted nasally into the trabecular meshwork and Schlemm's canal with a distance of 30 to $60^{\circ}$. The operating microscope is tilted $35^{\circ}$, while the head of the patient is turned $35^{\circ}$ counter-clockwise to ensure an optimal view into the chamber angle. The procedure includes a clear corneal incision using topical anesthesia. Usually topical anti-inflammatory and anti-infective medication is applied for 4 weeks postoperatively. 


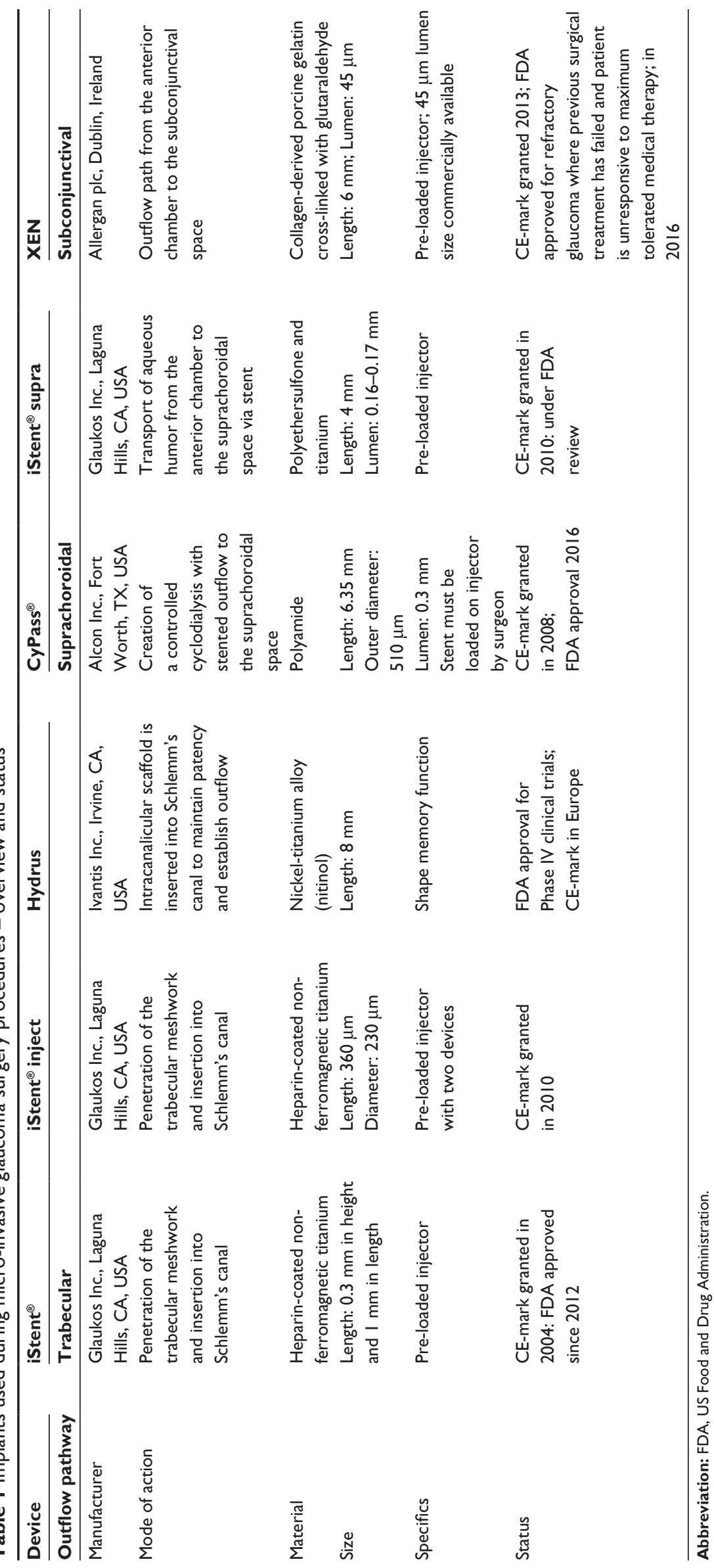


A

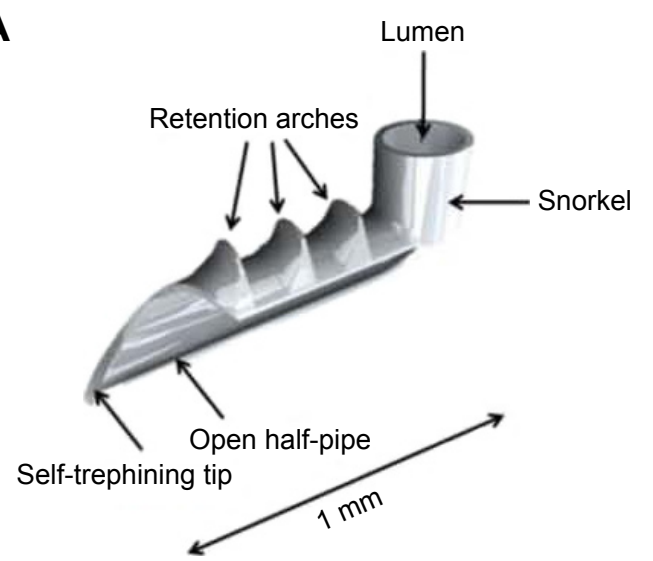

B

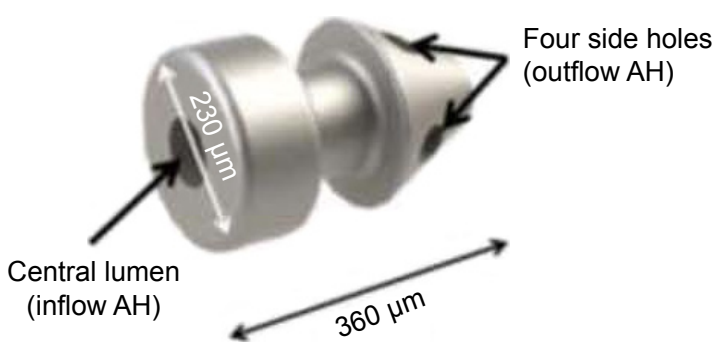

Figure I iStent ${ }^{\circledR}$ and iStent ${ }^{\circledR}$ inject.

Notes: The first generation iStent $(\mathbf{A})$ has a self-trephining tip which is inserted into Schlemm's canal. The device is maintained by the three retention arches. The lumen is directed toward the anterior chamber. (B) iStent inject possesses four side holes opening toward Schlemm's canal. The central lumen is directed toward the anterior chamber. Abbreviation: $\mathrm{AH}$, aqueous humour.

\section{Efficacy of iStent}

Efficacy and safety of iStent were evaluated in several clinical studies with different settings. The results of these studies are summarized in Tables 2 and 3 and Figures 2 and 3.

\section{Efficacy of iStent in combined procedures}

In most of the studies iStent was implanted during a combined procedure with cataract surgery including PE/IOL. In a randomized prospective study conducted by Fea, efficacy and safety of iStent combined with cataract surgery were compared with a stand-alone cataract surgery (PE/IOL) ${ }^{28}$ Mean medically treated baseline IOP (no wash-out phase) in the iStent study arm was $17.3 \pm 2.6 \mathrm{mmHg}$ compared to $17.3 \pm 3.0 \mathrm{mmHg}$ in the PE/IOL only study arm. At final visit at month 15 , mean IOP in the iStent arm was $14.8 \pm$ $1.2 \mathrm{mmHg}$ and $15.7 \pm 1.1 \mathrm{mmHg}$ in the $\mathrm{PE} / \mathrm{IOL}$ arm, respectively $(P=0.031)$. At month 16 after the wash-out of any study medications, mean IOPs increased to $16.6 \pm 3.1 \mathrm{mmHg}$ in the iStent arm and $19.2 \pm 3.5 \mathrm{mmHg}$ in the PE/IOL arm $(P=0.042)$. The mean number of medications used at baseline was $2.0 \pm 0.9$ in the iStent arm and $1.9 \pm 0.7$ in the PE/IOL arm compared to $0.4 \pm 0.7$ in the iStent and $1.3 \pm 1.0$ in the PE/IOL arm, respectively, at month $15(P=0.007)$.

In another prospective controlled and randomized study with 233 eyes with mild to moderate primary open-angle glaucoma (POAG), exfoliative and pigmentary glaucoma, and IOP values of $\leq 24 \mathrm{mmHg}$ treated with one to three medications at baseline, were randomized to receive either a stand-alone PE/IOL procedure $(\mathrm{n}=111)$ or a combined $\mathrm{PE} / \mathrm{IOL}$ procedure $(\mathrm{n}=122)$ with the implantation of an iStent. ${ }^{29}$ Mean baseline IOPs were $18.7 \pm 3.3 \mathrm{mmHg}$ (on medication) and $25.2 \pm 3.5 \mathrm{mmHg}$ (after wash-out) in the
iStent study arm, and $18.0 \pm 3.0 \mathrm{mmHg}$ (on medication) and 25.5 $\pm 3.7 \mathrm{mmHg}$ (after wash-out) in the PE/IOL study arm. Reduction of mean IOP at month 12 in comparison to mean baseline IOP after wash-out was similar in both arms, with a decrease of mean IOP of $8.4 \pm 3.6 \mathrm{mmHg}$ in the iStent arm and $8.5 \pm 4.3 \mathrm{mmHg}$ in the $\mathrm{PE} / \mathrm{IOL}$ arm, respectively. A significant difference at month 12 was seen in the number of medications used. Fifteen percent of the patients in the iStent arm received medications versus $35 \%$ in the $\mathrm{PE} / \mathrm{IOL}$ $\operatorname{arm}(P=0.001)$. Furthermore, $72 \%$ of patients in the iStent arm achieved IOP values of $\leq 21 \mathrm{mmHg}$ and an IOP reduction versus baseline of $\geq 20 \%$ compared to $50 \%$ of patients in the PE/IOL arm $(P<0.001)$. The safety profile of both arms was comparable.

In a prospective, randomized, controlled registration trial published by Craven et al efficacy and safety results of iStent were reported for a study period of 24 months. ${ }^{30}$ Two hundred and forty patients with mild to moderate glaucoma (POAG, exfoliative glaucoma [PEX], and pigmentary glaucoma) were randomized to $\mathrm{PE} / \mathrm{IOL}$ surgery alone $(n=123)$ or to the combined procedure with iStent $(\mathrm{n}=117)$. Mean baseline IOPs were 18.6 \pm 3.6 (treated) and $25.4 \pm 3.6 \mathrm{mmHg}$ (after wash-out) in the iStent arm,

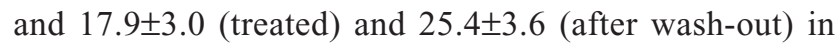
the PE/IOL arm, respectively. After 24 months mean IOPs decreased to $17.1 \pm 2.9$ in the iStent arm and 17.8 \pm $3.3 \mathrm{mmHg}$ in the $\mathrm{PE} / \mathrm{IOL}$ arm. Interestingly, 71 patients (61\%) in the iStent arm achieved IOP values, without medication, of $\leq 21 \mathrm{mmHg}$ versus only 61 patients (53\%) in the PE/IOL arm $(P=0.0036)$.

In a non-randomized interventional case-series published by Arriola-Villalobos et al data from 19 glaucoma patients who 
received one iStent during a combined cataract procedure were reported for a follow-up period of 5 years (mean: $53.7 \pm 9.3$ months). ${ }^{31}$ Mean IOP (without wash-out of medications) decreased significantly from $19.3 \pm 1.9 \mathrm{mmHg}$ at baseline to $16.3 \pm 4.2 \mathrm{mmHg}$ at final visit $(-16.3 \% ; P=0.002)$. The mean number of pressure-lowering medications decreased from $1.3 \pm 0.5$ at baseline to $0.8 \pm 0.9$ at final visit $(P=0.046)$.

In a prospective, non-randomized consecutive case series of 62 eyes with POAG, PEX, secondary glaucoma, posttraumatic glaucoma, and ocular hypertension which received iStent during a combined procedure with PE/IOL, Neuhann reported a decrease of mean IOP from $24.1 \pm 6.9 \mathrm{mmHg}$ on a mean of $1.8 \pm 0.9$ medications at baseline to $14.9 \pm 2.3 \mathrm{mmHg}$ at month 36. Medications were completely stopped in $74 \%$ of eyes at month $36 .{ }^{32}$

In a prospective case series conducted in the UK, iStent was implanted in 44 eyes with OAG either during a combined PE/IOL $(n=40)$ or a stand-alone procedure $(n=4) .{ }^{33}$ Mean IOP at baseline was $21.5 \mathrm{mmHg}$ and the mean number of medications used 2.3. At month 6 both, mean IOP and mean number of medications used, decreased significantly to $16.5 \mathrm{mmHg}$ $(P<0.001)$ and $0.5(P<0.001)$, respectively.

The effects of an implantation of multiple (two or three) iStents were evaluated in studies published by FernándezBarrientos et al, Belovay et al, Katz et al, Ahmed et al and Donnenfeld et al. ${ }^{34-38}$ This approach was further followed during the development of iStent inject which is delivered with two micro-stents in one injector.

In the study of Fernández-Barrientos et al the changes of aqueous humor dynamics were evaluated in patients with glaucoma or ocular hypertension undergoing a PE/IOL surgery. ${ }^{34}$ Patients of one study arm received two iStents together with a $\mathrm{PE} / \mathrm{IOL}$ procedure; patients of the second study arm received a $\mathrm{PE} / \mathrm{IOL}$ procedure only. In addition, IOP and the number of medications taken before and 12 months after the surgery were analyzed. Mean baseline IOPs were $24.2 \pm 1.8 \mathrm{mmHg}$ in the two-stent arm and $23.6 \pm 1.5 \mathrm{mmHg}$ in the PE/IOL only arm. At final visit, mean IOP decreased by $6.6 \mathrm{mmHg}$ in the two-stent arm to $17.6 \pm 2.8 \mathrm{mmHg}$, and by $3.8 \mathrm{mmHg}$ to $19.8 \pm 2.3 \mathrm{mmHg}(P=0.04)$ in the $\mathrm{PE} / \mathrm{IOL}$ arm, respectively. Aqueous outflow facility increased by $275 \%$ in the two-stent arm and by $46 \%$ in the PE/IOL arm at month 12 compared to baseline $(P<0.02)$. At baseline, mean number of medications used was comparable in both arms with $1.1 \pm 0.5$ in the two-stent arm and $1.2 \pm 0.7$ in the PE/IOL arm. No patient in the two-stent arm needed any medication at month 12, compared to a mean number of medications of $0.7 \pm 1.8$ in the PE/IOL arm $(P=0.007)$.
In a study published by Belovay et al two or three iStents were implanted in each of 53 individual eyes during a PE/ IOL surgery. ${ }^{35}$ Twenty-eight eyes received two iStents, 25 eyes received three iStents. Mean baseline IOPs were $17.3 \pm 4.0 \mathrm{mmHg}$ and $18.6 \pm 4.0 \mathrm{mmHg}$ in the two- and threestent treatment arms, respectively. At month 12, mean IOP decreased to $13.8 \mathrm{mmHg}$ and $14.8 \mathrm{mmHg}$ in the two- and three-stent arm, respectively. The difference was found not to be statistically significant. A significant difference was found in the mean number of medications used at final visit, which was reduced from 2.8 and 2.6 at baseline to 1.0 and 0.4 at final visit for the two- and three-iStent arms, respectively $(P<0.001)$. Overall, a $20 \%$ reduction of IOP with a reduction of medication of $64 \%$ was achieved in the two-iStent arm and a $20 \%$ reduction of IOP with a reduction of medications of $85 \%$ was achieved in the three-iStent arm.

In a retrospective consecutive case series conducted by Ferguson et al efficacy and safety of the iStent were evaluated in 350 eyes. The implantation of the iStent was performed during a combined procedure with a cataract extraction and phacoemulsification in patients with $\mathrm{OAG}$ and cataract. ${ }^{39}$ Mean medicated preoperative IOP was $19.13 \pm 6.34 \mathrm{mmHg}$. At 2 years after the procedure, mean IOP was significantly lower $(15.17 \pm 3.53 \mathrm{mmHg} ; P<0.0001)$ and the mean number of ocular hypotensive medications decreased from $1.19 \pm 1.0$ at baseline to $0.61 \pm 0.96$ at month $24(P<0.0001)$. An IOP level of $\leq 18 \mathrm{mmHg}$ at month 24 was achieved by $52 \%$ of the patients without medication.

Seibold et al evaluated the treatment outcomes of iStent (IOP, use of medication) in a retrospective case series of 46 eyes of 45 patients after combined phacoemulsification and the implantation of the trabecular micro-bypass (iStent) in patients with controlled glaucoma. ${ }^{40}$ Treatment success (defined as a $20 \%$ or more reduction in IOP or the discontinuation of at least one medication) was achieved in $76.1 \%$ of the patients at month 12 and $41 \%$ of patients were medication-free at month 12 . Overall mean IOP decreased from $14.7 \pm 3.2 \mathrm{mmHg}$ preoperatively to $13.2 \pm 2.8 \mathrm{mmHg}$ at month $12(P=0.01)$.

\section{Efficacy of iStent in stand-alone procedures}

Efficacy and safety of iStent implanted in stand-alone procedures without cataract surgery were evaluated in three clinical studies.

Katz et al evaluated the effect of the implantation of either one, two or three iStents during stand-alone procedures without PE/IOL in 119 patients with OAG. ${ }^{36}$ Thirty and eight subjects received one, 41 subjects two, and 40 subjects three iStents, respectively. At month 12 an unmedicated $\mathrm{IOP}$ of $\leq 18 \mathrm{mmHg}$ 
Table 2 iStent ${ }^{\circledR}$ and iStent ${ }^{\circledR}$ inject - overview of published study results

\begin{tabular}{lllll}
\hline Authors & $\begin{array}{l}\text { Study } \\
\text { design }\end{array}$ & $\begin{array}{l}\text { Eyes at } \\
\text { baseline }(\mathrm{n})\end{array}$ & Diagnoses & $\begin{array}{l}\text { Procedure } \\
\text { (number of stents) }\end{array}$
\end{tabular}

\begin{tabular}{|c|c|c|c|c|c|}
\hline \multicolumn{6}{|l|}{ iStent } \\
\hline \multicolumn{6}{|c|}{ iStent + CE (combined procedure) } \\
\hline $\mathrm{Fea}^{28}$ & $\mathrm{R}$ & 36 & POAG & iStent $(I)+C E$ & 15 \\
\hline Samuelson et $\mathrm{al}^{29}$ & $\mathrm{R}$ & 233 & POAG; PG; PEX & iStent $(I)+C E$ & 12 \\
\hline Craven et a ${ }^{30}$ & $\mathrm{R}$ & 240 & POAG; PG; PEX & iStent $(I)+C E$ & 24 \\
\hline Arriola-Villalobos et al ${ }^{31}$ & NR & 19 & POAG; PEX; PG & iStent $(I)+C E$ & 60 \\
\hline Neuhann ${ }^{32}$ & NR & 62 & $\mathrm{OAG} ; \mathrm{OH}$ & iStent $(I)+C E$ & 36 \\
\hline Patel et $\mathrm{al}^{33}$ & NR & 44 & $\begin{array}{l}\text { POAG; PEX; } \\
\text { other }\end{array}$ & $\begin{array}{l}\text { iStent }(I)+C E(n=40) \text {; } \\
\text { iStent only }(n=4)\end{array}$ & 6 \\
\hline Fernández-Barrientos et $\mathrm{al}^{34}$ & $\mathrm{R}$ & 33 & POAG; OH & iStent $(2)+C E$ & 12 \\
\hline Belovay et $\mathrm{a}^{35}$ & NR & 53 & $\begin{array}{l}\text { POAG; PEX; } \\
\text { other }\end{array}$ & iStent $(2$ or 3$)+C E$ & 12 \\
\hline Ferguson et a $\left.\right|^{39}$ & CS & 350 & $\begin{array}{l}\text { OAG (mild to } \\
\text { moderate) }\end{array}$ & iStent $(I)+C E$ & 24 \\
\hline Seibold et a ${ }^{40}$ & CS & 64 & POAG; other & iStent $(I)+C E$ & 12 \\
\hline \multicolumn{6}{|c|}{ iStent (stand-alone procedure) } \\
\hline Katz et $\mathrm{al}^{36}$ & $\mathrm{R}$ & 119 & $\begin{array}{l}\text { OAG including; } \\
\text { PEX and PG }\end{array}$ & $\begin{array}{l}\text { iStent }(I-3)(I): n=38 \\
\text { (2): } n=4 I ;(3): n=40\end{array}$ & 18 \\
\hline Ahmed et $\mathrm{al}^{37}$ & NR & 39 & OAG & iStent (2) & 18 \\
\hline Donnenfeld et $\mathrm{al}^{38}$ & NR & 76 & $\begin{array}{l}\text { OAG including; } \\
\text { PEX and PG }\end{array}$ & iStent (2) & 36 \\
\hline
\end{tabular}




\begin{tabular}{|c|c|c|c|c|}
\hline $\begin{array}{l}\text { Mean IOP at } \\
\text { baseline }(\mathrm{mmHg}) \\
\text { medicated }(\mathrm{M}) \text { or } \\
\text { unmedicated }(\mathrm{U})\end{array}$ & $\begin{array}{l}\text { Decrease of mean IOP } \\
\mathrm{mmHg}(\%)[\text { month] }\end{array}$ & $\begin{array}{l}\text { Reduction of } \\
\text { number of } \\
\text { medications } \\
\text { (mean) } \\
\text { [month] }\end{array}$ & $\begin{array}{l}\text { Wash-out phase } \\
\text { for medication }\end{array}$ & Study details/remarks \\
\hline
\end{tabular}

\begin{tabular}{|c|c|c|c|c|}
\hline $17.9(\mathrm{M})$ & $3.2(17.3)[15]$ & $1.6[15]$ & Yes & $\begin{array}{l}\text { Primary efficacy outcome: IOP. } \\
\text { Secondary outcome measure: Number } \\
\text { and type of glaucoma medications }\end{array}$ \\
\hline I8.4 (M); 25.4 (U) & $8.4(33.0)[12]$ & $1.4[12]$ & Yes & $\begin{array}{l}\text { Primary efficacy endpoint: \% subjects } \\
\text { with IOP } \leq 2 \mathrm{ImHg}\end{array}$ \\
\hline I8.6 (M); $25.4(\mathrm{U})$ & $\begin{array}{l}8.4(33.1)[12] \\
8.5(33.5)[24]\end{array}$ & $\begin{array}{l}\mathrm{I} .4[12] \\
\mathrm{I} .3[24]\end{array}$ & Yes & $\begin{array}{l}\text { Primary efficacy endpoint: \% subjects } \\
\text { with IOP } \leq 2 \mathrm{I} \mathrm{mmHg} \text { at month } \mathrm{I} 2 \text {. } \\
\text { Secondary efficacy endpoint: \% subjects } \\
\text { with IOP reduction } \geq 20 \% \text { without } \\
\text { medication at month } \mathrm{I} 2\end{array}$ \\
\hline $19.42(M)$ & $3.34(17.2)[60]$ & $\begin{array}{l}\text { I.I5 [I2]; } \\
\text { I.0 [24]; } 0.76[36] ; \\
0.82[48] ; 0.17[60]\end{array}$ & No & $\begin{array}{l}\text { Primary efficacy outcome: IOP. } \\
\text { Secondary outcome measure: Number } \\
\text { and type of glaucoma medications }\end{array}$ \\
\hline $24 . I(M)$ & $\begin{array}{l}9.3(38.6)[12] \\
9.6(39.8)[24] \\
9.2(38.2)[36]\end{array}$ & $\begin{array}{l}\text { I.3 [I2]; I.6 [24]; } \\
\text { I.5 [36] }\end{array}$ & No & $\begin{array}{l}\text { Efficacy outcome measures: IOP and } \\
\text { number of glaucoma medications }\end{array}$ \\
\hline $21.5(M)$ & $5.0(23.3)[6]$ & $1.71[12]$ & No & $\begin{array}{l}\text { Efficacy outcome measures: IOP and } \\
\text { number of glaucoma medications }\end{array}$ \\
\hline $24.2(U)$ & $\begin{array}{l}8.6(35.5)[6] \\
6.6(27.3)[12]\end{array}$ & I.I [12] & Yes & $\begin{array}{l}\text { Efficacy outcome measures: IOP and } \\
\text { number of glaucoma medications; } \\
\text { Aqueous flow rate, trabecular } \\
\text { outflow facility }\end{array}$ \\
\hline $\begin{array}{l}\text { Two-stent: } 17.3(\mathrm{M}) \\
\text { Three-stent: } 18.6(\mathrm{M})\end{array}$ & $\begin{array}{l}\text { Two-stent: } 3.5(20.2 \%)[12] \\
\text { Three-stent: } 3.8(20.4)[12]\end{array}$ & $\begin{array}{l}\text { Two-stent: } 1.8 \\
\text { Three-stent: } 2.2\end{array}$ & No & $\begin{array}{l}\text { Efficacy outcome measures: IOP and } \\
\text { number of glaucoma medications }\end{array}$ \\
\hline 19.13 (M) & $\begin{array}{l}3.54(18.5)[12] \\
3.96(20.7)[24]\end{array}$ & $\begin{array}{l}0.61[12] \\
0.58[24]\end{array}$ & No & $\begin{array}{l}\text { Primary efficacy endpoint: Development } \\
\text { of IOP. Secondary endpoint: Number } \\
\text { and type of ocular hypotensive } \\
\text { medications }\end{array}$ \\
\hline 14.7 (M) & $1.5(10.2)[12]$ & $0.4[12]$ & No & $\begin{array}{l}\text { Efficacy outcome measures: IOP and } \\
\text { number of glaucoma medications, } \\
\text { success and failure }\end{array}$ \\
\hline
\end{tabular}

\begin{tabular}{|c|c|c|c|c|}
\hline One-stent: 19.8 (M); & I0.I (40.4) [12-13]; & Decrease of mean & Yes & Primary efficacy endpoint: \% subjects \\
\hline $25.0(U)$ Two-stent: & II.4 (45.6) [12-13]; & IOP shown for & & with $I O P$ reduction $\geq 20 \%$ without \\
\hline 20.1 (M); $25.0(U)$ & $12.4(49.4)[12-13]$ & patients without & & medication at month 12 . Secondary \\
\hline Three-stent: 20.4 & & medication at & & efficacy endpoints: \% subjects with \\
\hline \multirow[t]{2}{*}{ (M); $25 . I$ (U) } & & month 18 & & $\mathrm{IOP} \leq 18 \mathrm{mmHg}$ \\
\hline & & & & $\begin{array}{l}\text { Further additional efficacy measure: } \\
\text { mean IOP and mean IOP reduction }\end{array}$ \\
\hline \multirow[t]{2}{*}{22.2 (M); $25.3(\mathrm{U})$} & $8.2(32.4)[13]$ & 1.0 & Yes & Pnmary efficacy endpoint: \% subjects with \\
\hline & & & & $\begin{array}{l}\text { IOP reduction } \geq 20 \% \text { with reduction of } \\
\text { one medication at month } \mathrm{I} 2 \text {. Secondary } \\
\text { efficacy endpoint: \% subjects with IOP } \\
\text { reduction } \leq 18 \mathrm{mmHg} \text { with reduction of } \\
\text { one medication at month } 12\end{array}$ \\
\hline \multirow[t]{4}{*}{20.6 (M); $24 . I(U)$} & $10.6(44.0)[12]$ & $35 / 39$ eyes $(89.7 \%)$ & Yes & Primary efficacy endpoint: \\
\hline & $10.6(44.0)[24]$ & did not require & & Month 12 IOP reduction $\geq 20 \%$ without \\
\hline & $8.9(36.9)[36]$ & ocular hypotensive & & medication. Secondary efficacy endpoint: \\
\hline & & medications & & Month 12 IOP reduction $\leq 18 \mathrm{mmHg}$ \\
\hline
\end{tabular}


Table 2 (Continued)

\begin{tabular}{lllll}
\hline Authors & $\begin{array}{l}\text { Study } \\
\text { design }\end{array}$ & $\begin{array}{l}\text { Eyes at } \\
\text { baseline }(n)\end{array}$ & Diagnoses & $\begin{array}{l}\text { Procedure } \\
\text { (number of stents) }\end{array}$
\end{tabular}

\begin{tabular}{llllll}
\hline iStent inject & & & & \\
\hline Voskanyan et al $^{17}$ & NR & 99 & POAG; PG; PEX & iStent inject (2)
\end{tabular}

$\begin{array}{lllll}\text { Klamann et al }^{41} & \text { CS } & 35 & \text { POAG; PEX; PG } & \text { iStent inject (2) } \\ \text { Fea et al }{ }^{42} & \text { R } & 94 & \text { OAG } & \text { iStent inject (2) versus } \\ \text { FC PGA/TIM }\end{array}$

Notes: Numbers in square brackets indicate months of follow-up. iStent, iStent inject - Glaukos Inc., Laguna Hills, CA, USA.

Abbreviations: IOP, intraocular pressure; OAG, open-angle glaucoma; POAG, primary open-angle glaucoma; PG, pigmentary glaucoma; PEX, exfoliative glaucoma; OH, ocular hypertension; R, randomized study; NR, non-randomized study; CS, case series; CE, cataract extraction; FC PGA/TIM, fixed combination prostaglandin-analog/timolol.

and an IOP reduction of $\geq 20 \%$ without ocular hypotensive medication were achieved by $89.2 \%, 90.2 \%$, and $92.1 \%$ of eyes in the one-, two-, and three-stent subgroups, respectively. At month 18, a medication was required by seven subjects $(18.4 \%)$ in the one-stent subgroup, four subjects $(9.8 \%)$ in the two-stent subgroup, and three subjects (7.5\%) in the three-stent subgroup, respectively. At month 18, mean unmedicated IOPs were $15.9 \pm 0.9 \mathrm{mmHg}, 14.1 \pm 1.0 \mathrm{mmHg}$, and $12.2 \pm 1.1 \mathrm{mmHg}$ for the one-, two-, and three-stent subgroups, respectively.

The IOP lowering effect of the implantation of two trabecular micro-bypass stents (iStent) and postoperative medical treatment with a prostaglandin-analog (travoprost) in patients with OAG, which was preoperatively uncontrolled with two ocular hypotensive medications, was evaluated in a non-randomized, prospective open-label study by Ahmed et $\mathrm{al}^{39}$ Overall, 39 phakic patients with medicated IOP between $18 \mathrm{mmHg}$ and $30 \mathrm{mmHg}$ and baseline IOP levels between $22 \mathrm{mmHg}$ and $38 \mathrm{mmHg}$ received two iStents and postoperative medical treatment with travoprost. Follow-up visits were scheduled over 18 months and a wash-out of medications was performed postoperatively at month 13 .
All patients achieved an IOP reduction of $\geq 20 \%$ and target IOP levels of $\leq 18 \mathrm{mmHg}$ at month 12 . At month 18 , mean medicated IOP decreased from 22.2 $\pm 2.0 \mathrm{mmHg}$ at baseline on two medications to $13.0 \pm 2.4 \mathrm{mmHg}$ on one medication. The mean unmedicated IOP decreased from $25.3 \pm 1.8 \mathrm{mmHg}$ preoperatively to $17.1 \pm 2.2 \mathrm{mmHg}$ at month 13 .

Donnenfeld et al examined efficacy and safety of the implantation of two iStents in phakic or pseudophakic patients with OAG and IOP levels between $18 \mathrm{mmHg}$ and $30 \mathrm{mmHg}$ on one preoperative ocular hypotensive medication over a period of 36 months. ${ }^{40}$ The primary efficacy endpoint, which was defined as a reduction of IOP of $\geq 20 \%$ from unmedicated baseline IOP at month 12, was achieved in 36 of 39 eyes $(92.3 \%)$. At month 36, mean IOP decreased significantly by $9.1 \pm 2.7 \mathrm{mmHg}(P<0.001)$ or $37 \%$ versus unmedicated baseline IOP, and by $5.5 \pm 2.7 \mathrm{mmHg}(P<0.001)$ or $26 \%$ versus medicated baseline IOP.

\section{Efficacy of iStent inject}

iStent inject is a micro-stent system which uses the smallest of all micro-stents available (Figure 1). Two of these 


\begin{tabular}{|c|c|c|c|c|}
\hline $\begin{array}{l}\text { Mean IOP at } \\
\text { baseline }(\mathrm{mmHg}) \\
\text { medicated }(\mathrm{M}) \text { or } \\
\text { unmedicated }(\mathrm{U})\end{array}$ & $\begin{array}{l}\text { Decrease of mean IOP } \\
\mathrm{mmHg}(\%) \text { [month] }\end{array}$ & $\begin{array}{l}\text { Reduction of } \\
\text { number of } \\
\text { medications } \\
\text { (mean) } \\
\text { [month] }\end{array}$ & $\begin{array}{l}\text { Wash-out phase } \\
\text { for medication }\end{array}$ & Study details/remarks \\
\hline $26.3(\mathrm{U}) 22.1(\mathrm{M})$ & $\begin{array}{l}10.6(40.3)[12] \\
6.4(29.0)[12]\end{array}$ & $\begin{array}{l}\text { No medication: } \\
71.7 \%[12] \text {; I5.2\% } \\
\text { with reduction on } \\
\text { one medication } \\
\text { [I2]; } 53.5 \% \text { with } \\
\text { reduction on } \\
\text { two medications } \\
\text { [12]; I7.2\% with } \\
\text { reduction on } \\
\text { three medications } \\
\text { [12]; I\% }\end{array}$ & Yes & $\begin{array}{l}\text { Primary efficacy endpoint: Month } 12 \\
\text { IOP } \leq \text { I } 8 \mathrm{mmHg} \text { without medication; } \\
\text { Secondary efficacy endpoint: Month } \\
\text { I } 2 \text { IOP } \leq 18 \mathrm{mmHg} \text { regardless of } \\
\text { medications }\end{array}$ \\
\hline $\begin{array}{l}\text { POAG: } 21.19(\mathrm{M}) \\
\text { PEX: } 23.75(\mathrm{M})\end{array}$ & $\begin{array}{l}\text { POAG: } 7.0(33.0) \text { [6]; } \\
\text { PEX: } 8.42(35.5)[6]\end{array}$ & $\begin{array}{l}\text { POAG: I.3I; } \\
\text { PEX: I. } 29\end{array}$ & No & $\begin{array}{l}\text { Primary efficacy endpoint: \% decrease } \\
\text { from medicated baseline IOP at month } \\
6 \text { in subgroups of patients with POAG, } \\
\text { PEX and PG }\end{array}$ \\
\hline $\begin{array}{l}\text { iStent inject arm: } \\
21 . I(M) 25.2(U) \\
\text { Two medication arm: } \\
20.7(M) 24.8(U)\end{array}$ & $\begin{array}{l}\text { iStent inject arm: } 12.2 \\
\text { (48.4) [12] } \\
\text { Two medication arm: } 11.6 \\
\text { (53.2) [12] }\end{array}$ & $\begin{array}{l}\text { Four patients on } \\
\text { medication at } \\
\text { month } 12 \text { in iStent } \\
\text { inject arm }\end{array}$ & Yes & $\begin{array}{l}\text { Patients enrolled uncontrolled on one } \\
\text { medication Primary efficacy endpoint: } \\
\% \text { subjects with IOP reduction } \geq 20 \% \\
\text { without medication. Secondary } \\
\text { efficacy endpoints: \% subjects with } \\
\text { IOP } \leq 18 \mathrm{mmHg} \text {; mean IOP and mean } \\
\text { IOP reduction }\end{array}$ \\
\hline
\end{tabular}

micro-devices are delivered in one injector, and it offers the convenient option to implant both micro-stents during the same procedure into the trabecular meshwork. In all published clinical studies, iStent inject was used during stand-alone procedures in phakic or pseudophakic patients. The results of these studies are summarized in Table 2 and Figure 4.

In a study published by Voskanyan et al two iStent inject were implanted in each of 99 individual eyes. ${ }^{17}$ Mean baseline IOPs were $22.1 \pm 1.3 \mathrm{mmHg}$ (with medication) and $26.3 \pm 3.5 \mathrm{mmHg}$ (after wash-out). At month 12, IOP decreased by $40.2 \%$ versus baseline to $15.7 \pm 3.7 \mathrm{mmHg}$. Of the patients, $24.4 \%$ required a medical treatment. IOP $\leq 18 \mathrm{mmHg}$ with and without medication was achieved by $81 \%$ and $66 \%$ of the patients, respectively. An IOP decrease of $\geq 20 \%$ and $\geq 30 \%$ without medication versus baseline was achieved by $72 \%$ and $61 \%$ of the patients, respectively. At month $12,86.9 \%$ of subjects had reduced their number of medications: in $15.2 \%$ and $71.7 \%$ of subjects the number of medications was reduced by one, and two or more medications, respectively.

Another study on the implantation of two iStent inject in each eye during stand-alone procedures without $\mathrm{PE} / \mathrm{IOL}$ was reported by Klamann et al. ${ }^{41}$ In this study, 17 patients with POAG, 15 patients with PEX, and three patients with pigmentary glaucoma were included. In the subgroup of patients with POAG, mean IOP decreased significantly from 21.2 \pm $2.6 \mathrm{mmHg}$ to $14.2 \pm 1.3 \mathrm{mmHg}$ after 6 months and the mean number of medications used decreased from $2.2 \pm 0.9$ at baseline to $0.9 \pm 0.6$ at month 6 . In the patient subgroup with PEX, mean IOP was significantly reduced from $23.8 \pm 3.2 \mathrm{mmHg}$ at baseline to $15.3 \pm 1.1 \mathrm{mmHg}$ at month 6 , and the mean number of medications was reduced from $2.3 \pm 1.2$ at baseline to $1.0 \pm$ 0.3 at month 6. In patients with POAG and PEX, mean number of medications was reduced by -1.3 and -1.29 at month 6 .

Finally, iStent inject was evaluated in comparison with medical therapy using a fixed combination of a prostaglandin and timolol, by Fea et $\mathrm{al}^{42}$ Ninety and four and 98 eyes were treated with the implantation of two iStent inject or a fixed combination of latanoprost and timolol or travoprost and timolol, respectively. Mean baseline IOPs in the iStent inject and medication study arms were $26.3 \pm 2.5 \mathrm{mmHg}$ and $24.8 \pm 2.7 \mathrm{mmHg}$, respectively. Decrease of IOP was comparable in both treatment arms. At month 12, mean 
Table 3 iStent ${ }^{\circledR}$ and iStent ${ }^{\circledR}$ inject: proportion of patients achieving target IOP levels of $\leq 18 \mathrm{mmHg}$ or $\leq 2 \mathrm{I} \mathrm{mmHg}$ and/or an IOP reduction of $\geq 20 \%$ versus baseline IOP levels

\begin{tabular}{|c|c|c|c|c|c|}
\hline Authors & $\begin{array}{l}\text { Number } \\
\text { of iStents } \\
\text { implanted }\end{array}$ & $\begin{array}{l}\% \text { subjects with IOP } \leq 18 \mathrm{mmHg} \\
\text { or } \leq 2 \mathrm{I} \mathrm{mmHg} \text { without ocular } \\
\text { hypertensive medication }\end{array}$ & $\begin{array}{l}P \text {-value or } \\
95 \% \mathrm{Cl}\end{array}$ & $\begin{array}{l}\% \text { subjects with IOP } \\
\text { reduction } \geq 20 \% \text { without } \\
\text { ocular hypertensive } \\
\text { medication }\end{array}$ & $\begin{array}{l}P \text {-value or } \\
95 \% \mathrm{Cl}\end{array}$ \\
\hline \multicolumn{6}{|l|}{ iStent } \\
\hline \multicolumn{6}{|c|}{ iStent + CE (combined procedure) } \\
\hline Samuelson et al ${ }^{29}$ & I & $\begin{array}{l}\leq 21 \mathrm{mmHg}: \text { iStent + CE: } 72 \%[12] \\
\leq 21 \mathrm{mmHg}: \text { CE alone: } 50 \%[12]\end{array}$ & $P<0.001$ & $\begin{array}{l}\text { iStent + CE: } 66 \%[12] \\
\text { CE: } 48 \%[12]\end{array}$ & $P=0.003$ \\
\hline Craven et $\mathrm{al}^{30}$ & I & $\begin{array}{l}\leq 21 \mathrm{mmHg}: \text { iStent + CE: 7I\% [24] } \\
\leq 21 \mathrm{mmHg}: \text { CE: } 61 \% \text { [24] }\end{array}$ & $P=0.036$ & $\begin{array}{l}\text { iStent + CE: } 61 \%[24] \\
\text { CE: } 54 \%[24]\end{array}$ & $P=0.090$ \\
\hline Arriola-Villalobos et al ${ }^{\mid 1}$ & I & $\begin{array}{l}\leq 21 \mathrm{mmHg}: \text { iStent + CE: } 42.1 \%[60]^{*} \\
\leq 21 \mathrm{mmHg}: \text { iStent + CE: } 89.5 \%[60]^{* *}\end{array}$ & na & na & na \\
\hline Ferguson et $\mathrm{al}^{39}$ & I & $\leq 18$ mmHg: iStent + CE: 52.0\% [24] & na & na & na \\
\hline Seibold et al ${ }^{40}$ & I & na & na & iStent + CE: 76.1\% [I2] & $62.4 \%-90.0 \%$ \\
\hline \multicolumn{6}{|c|}{ iStent (stand-alone procedure) } \\
\hline Katz et $\mathrm{al}^{36}$ & $\begin{array}{l}1 \\
2 \\
3\end{array}$ & $\begin{array}{l}\leq 18 \mathrm{mmHg}: 89.2 \%[12] \\
\leq 18 \mathrm{mmHg}: 90.2 \%[\mathrm{I} 2] \\
\leq 18 \mathrm{mmHg}: 92.1 \%[12]\end{array}$ & $\begin{array}{l}74.6 \%-97.0 \% \\
76.9 \%-97.3 \% \\
78.6 \%-98.3 \%\end{array}$ & $\begin{array}{l}89.2 \%[12] \\
90.2 \%[12] \\
92.1 \%[12]\end{array}$ & $\begin{array}{l}74.6 \%-97.0 \% \\
76.9 \%-97.3 \% \\
78.6 \%-98.3 \%\end{array}$ \\
\hline Ahmed et al ${ }^{37}$ & 2 & $\leq 18 \mathrm{mmHg}: 100.0 \%[12]^{* * *}$ & na & $100.0 \%[12]^{*}$ & na \\
\hline Donnenfeld et $\mathrm{al}^{38}$ & 2 & $\begin{array}{l}\leq 18 \mathrm{mmHg}: 92.3 \%[12] \\
\leq 18 \mathrm{mmHg}: 89.7 \%[36]\end{array}$ & $\begin{array}{l}79.1 \%-98.4 \% \\
72.6 \%-97.8 \%\end{array}$ & $\begin{array}{l}92.3 \%[12] \\
86.2 \%[36]\end{array}$ & $\begin{array}{l}79.1 \%-98.4 \% \\
68.3 \%-96.1 \%\end{array}$ \\
\hline \multicolumn{6}{|l|}{ iStent inject } \\
\hline Voskanyan et al ${ }^{17}$ & 2 & $\begin{array}{l}\leq 18 \mathrm{mmHg}: 66.0 \%[12]^{*} \\
\leq 18 \mathrm{mmHg}: 81.0 \%[12]^{* *}\end{array}$ & $\begin{array}{l}55 \%-76 \% \\
71 \%-88 \%\end{array}$ & $\begin{array}{l}72 \%[12] \\
93 \%[12]\end{array}$ & $\begin{array}{l}61 \%-81 \% \\
86 \%-97 \%\end{array}$ \\
\hline Fea et $\mathrm{al}^{42}$ & 2 & $\begin{array}{l}\text { iStent: } \leq 18 \mathrm{mmHg}: 92.6 \%[12] \\
\text { PGA/TIM: } \leq 18 \mathrm{mmHg}: 89.7 \%[12]\end{array}$ & $\begin{array}{l}85.3 \%-97.0 \% \\
82.0 \%-95.0 \%\end{array}$ & $\begin{array}{l}\text { iStent: } 94.7 \%[12] \\
\text { PGA/TIM: } 91.8[12]\end{array}$ & $\begin{array}{l}88.0 \%-98.3 \% \\
84.5 \%-96.4 \%\end{array}$ \\
\hline $\begin{array}{l}\text { Notes: iStent, iStent inject } \\
\text { and Voskanyan et a }\left.\right|^{17} \text { the pr } \\
\text { medication, } * * \text { all patients, irr } \\
\text { is shown. }\end{array}$ & $\begin{array}{l}\text { aukos Inc., } \\
\text { tion of patie } \\
\text { ctive of med }\end{array}$ & $\begin{array}{l}\text { Hills, CA, USA. Numbers in square bra } \\
\text { ith IOP values of } \leq 18 \mathrm{mmHg} \text { is given sep } \\
\text { at final visit. ***/n the study of Ahmed et }\end{array}$ & $\begin{array}{l}\text { ndicate months } \\
\text { at final visit for }\end{array}$ & $\begin{array}{l}\text { ollow-up. In the studies of } \mathrm{Ar} \\
\text { patients regardless of patients } \\
\text { ects with a maximum of one } \mathrm{n}\end{array}$ & $\begin{array}{l}\text { Villalobos et a }\left.\right|^{31} \\
\text { cation: *without } \\
\text { ation at final visit }\end{array}$ \\
\hline
\end{tabular}

IOP decreased to $13.0 \pm 2.3 \mathrm{mmHg}$ in the iStent arm and to $13.2 \pm 2.0 \mathrm{mmHg}$ in the medication arm. Four patients in the iStent arm were taking medications at month 12 .

\section{Safety profile and adverse events of iStent and iStent inject}

Blood reflux from Schlemm's canal into the anterior chamber is a common process that occurs intraoperatively. This reflux may be seen as a positive and normal sign which occurs when iStent and iStent inject are well positioned in the trabecular meshwork. The most common adverse events in all studies were minor, and include temporary obstructions of the iStent, which were resolved in most cases by Neodym-YAG laser treatment, and malpositioned micro-stents. No postoperative hypotony, loss of endothelial cells, and signs of inflammation were reported in any of the studies. As for all trabecular procedures, caution should be applied in patients with elevated EVP, ie, in patients with lower baseline IOPs and in patients with obesity or metabolic syndrome.

\section{Hydrus ${ }^{\circledR}$ micro-stent}

The Hydrus micro-stent is a so-called "intracanalicular scaffold"; an $8 \mathrm{~mm}$ long slightly shaped device (Table 1) which is implanted inside Schlemm's canal. The micro-stent is implanted $\mathrm{ab}$ interno with a pre-loaded injector through a clear corneal incision into Schlemm's canal. After the implantation, the Hydrus micro-stent dilates Schlemm's canal in the complete nasal quadrant, allowing aqueous humor to bypass the trabecular meshwork through multiple collector channels (Figure 5).

\section{Surgical procedure with the Hydrus micro-stent}

The Hydrus micro-stent is implanted in the nasal hemisphere or the inferior temporal quadrant through a 1-1.5 mm clear corneal incision. The beveled tip of the injector is used to perforate the trabecular meshwork, and to position the micro-stent in Schlemm's canal by rotating the advancement mechanism. Usually acetylcholine is recommended to establish a miosis, and a viscoelastic substance is used to stabilize the anterior 


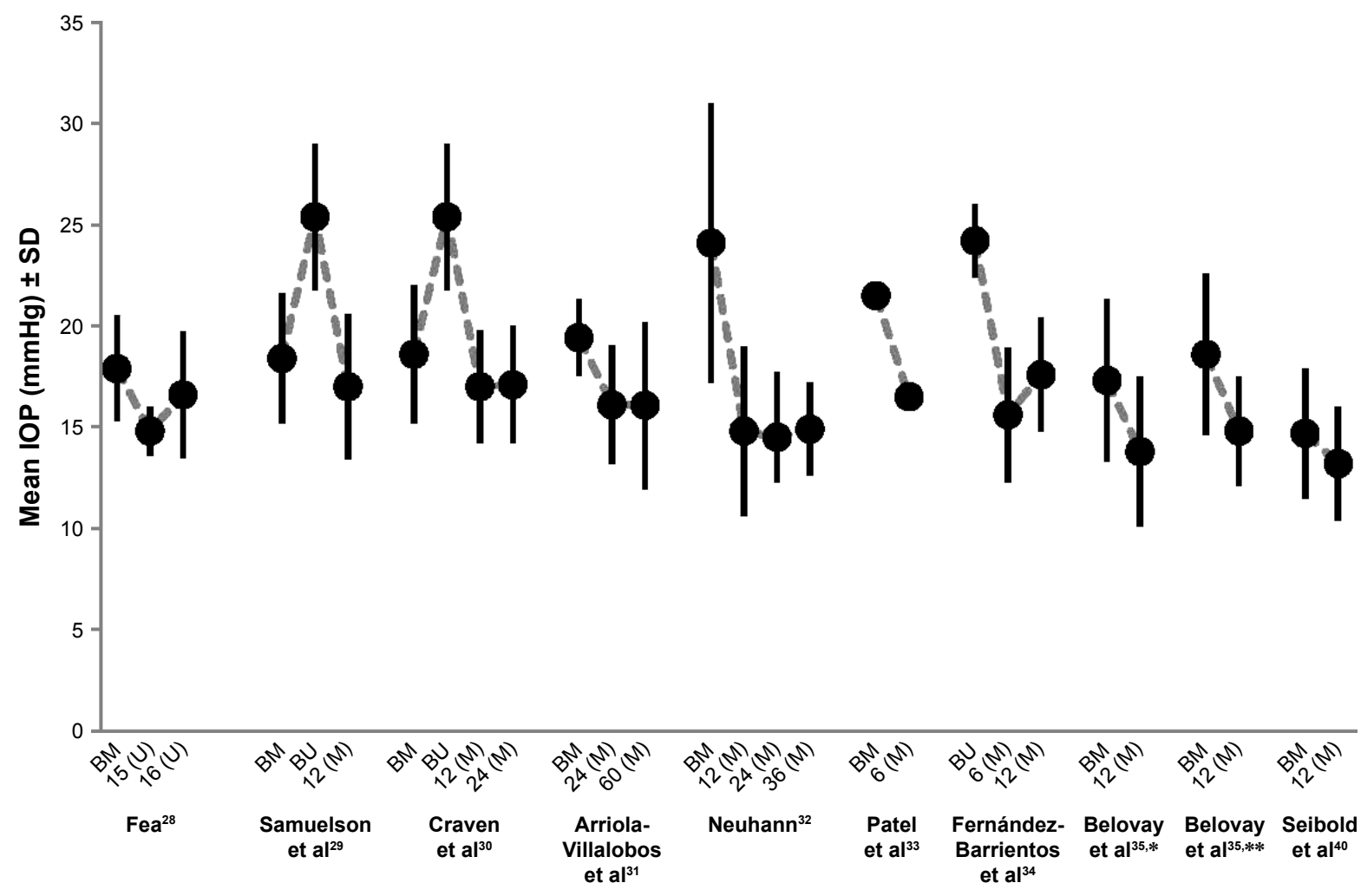

Figure 2 Development of mean IOP after the implantation of iStent ${ }^{\circledR}$ during combined procedures (implantation with phacoemulsification and IOL implantation). Notes: Reduction of mean intraocular pressure compared to medicated and/or unmedicated baseline visit. In the study of Belovay et al ${ }^{35}$ the results were reported separately for patients with the implantation of two or three iStents (*one iStent, ${ }^{*}$ two iStents implanted). In the study by Patel et al ${ }^{33}$ no standard deviations were given. Numbers on the horizontal axis indicate the month of visit.

Abbreviations: IOP, intraocular pressure; IOL, intraocular lens; BM, medicated baseline IOP; BU, unmedicated baseline IOP; U, unmedicated; M, medicated.

chamber. At the end of the procedure the viscoelastic substance is removed and the anterior chamber inflated with balanced salt solution. A topical antibiotic and a topical corticosteroid are recommended during the first postoperative period.

Pfeiffer et al reported results of a controlled, prospective, randomized, single-masked 2-year clinical study comparing the efficacy and safety of a combined procedure with the Hydrus micro-stent and cataract surgery using PE/IOL and cataract surgery alone. ${ }^{18}$

\section{Efficacy of Hydrus}

In this study, prior glaucoma medications were discontinued and washed-out before baseline IOP measurements were conducted. Mean baseline IOPs were $26.3 \pm 4.4 \mathrm{mmHg}$ in the Hydrus/PE/IOL study arm and $26.6 \pm 4.2 \mathrm{mmHg}$ in the PE/ IOL study arm. At the 24-month follow-up visit, mean IOPs were $16.9 \pm 3.3 \mathrm{mmHg}$ in the Hydrus/PE/IOL study arm and $19.2 \pm 4.7 \mathrm{mmHg}$ in the PE/IOL study arm. At 24 months, a $20 \%$ reduction from baseline IOP was achieved by $80 \%$ of patients treated with Hydrus/PE/IOL as compared to $46 \%$ of patients treated with PE/IOL only. The percentage of unmedicated patients at month 24 was significantly higher in the Hydrus/PE/IOL study arm (72.9\%) compared to the PE/IOL study arm (37.8\%).

\section{Safety profile and adverse events of Hydrus}

Forty-seven out of 50 patients in the Hydrus/PE/IOL study arm and 43 patients in the PE/IOL study arm were evaluable at month 24. Significantly more patients in the Hydrus/PE/ IOL study arm (12\%) developed focal peripheral anterior synechiae, typically present as focal iris tissue adhesion to the device or to the chamber angle and located at or near the inlet of the Hydrus micro-stent. Interestingly the presence of these synechiae seemed not to have any influence on the study outcome. Other adverse events were similarly distributed among the two study arms.

\section{Procedures targeting the suprachoroidal space CyPass ${ }^{\circledR}$ micro-stent}

The CyPass micro-stent (Alcon Inc., Fort Worth, TX, USA) creates an outflow pathway from the anterior chamber to the suprachoroidal space (Table 1). The device, with a length of $6.35 \mathrm{~mm}$, is placed through a clear corneal incision after 


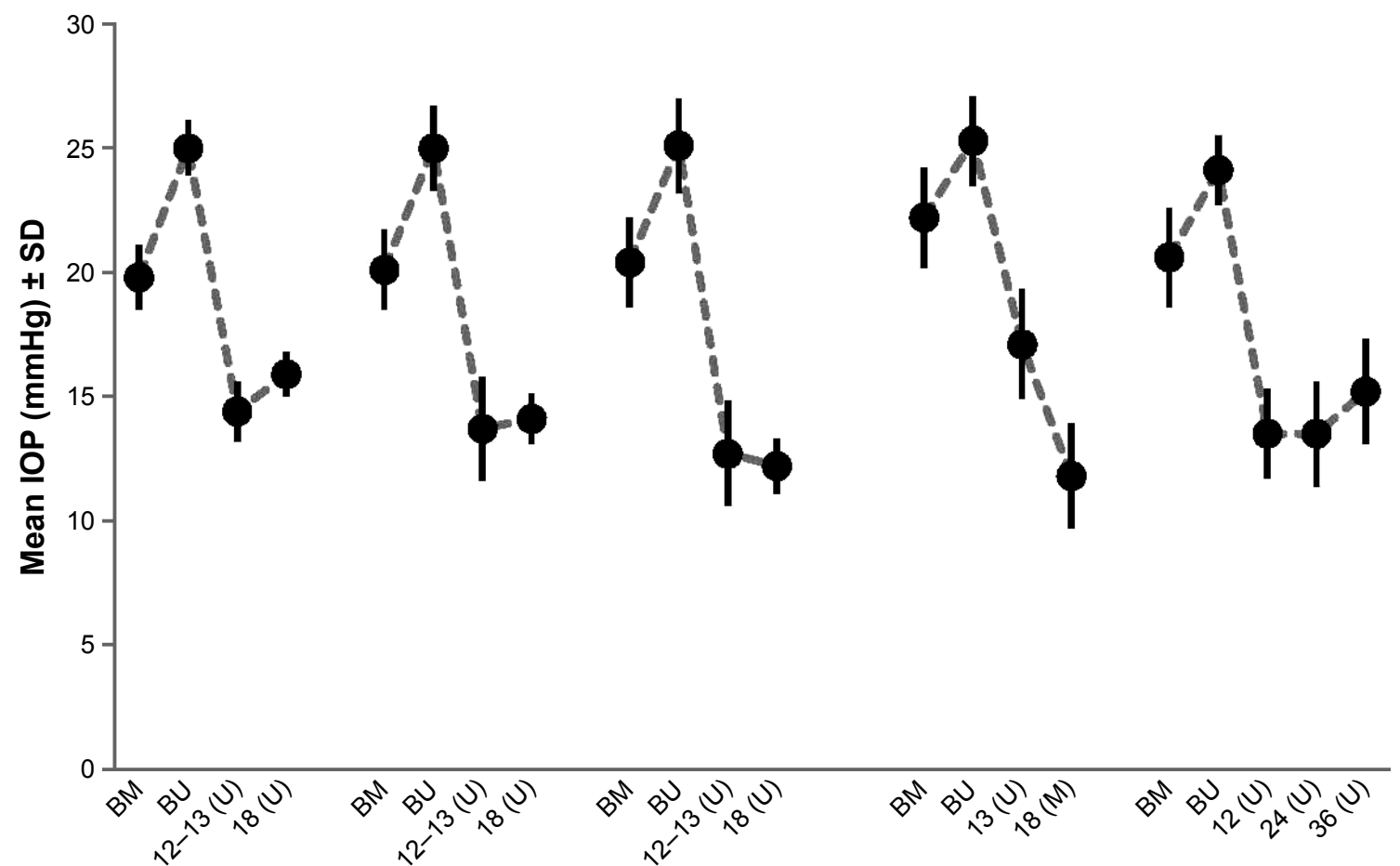

One iStent

Two iStents,

Three iStents

Ahmed et $\mathbf{a l}^{37}$

Figure 3 Development of mean IOP after the implantation of iStent ${ }^{\circledR}$ during stand alone procedures.

Notes: Reduction of mean intraocular pressure at last visit compared to medicated and/or unmedicated baseline visit. In the study of Katz et al ${ }^{36}$ the results were reported separately for patients with the implantation of one, two or three iStents. In the study of Ahmed et al ${ }^{39}$ IOP lowering medication (travoprost) was re-established at month 13. Month 18 represents the IOP data including travoprost as a medical therapy. Numbers on the horizontal axis indicate the month of visit.

Abbreviations: IOP, intraocular pressure; BM, medicated baseline IOP; BU, unmedicated baseline IOP; U, unmedicated; M, medicated.

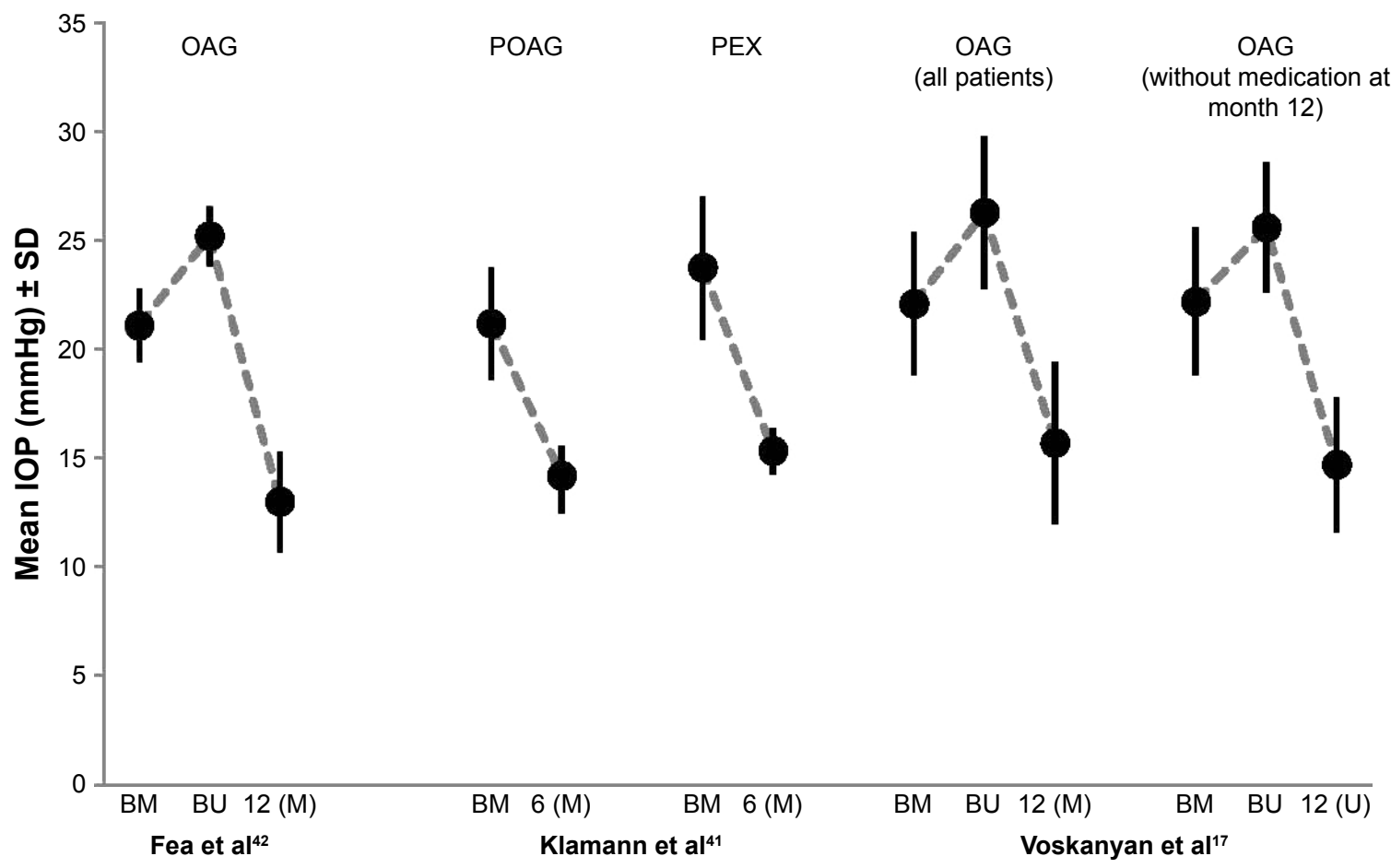

Figure 4 Development of mean IOP after the implantation of iStent inject ${ }^{\circledR}$ during stand alone procedures.

Notes: Reduction of mean intraocular pressure at last visit compared to medicated and/or unmedicated baseline visit. iStent inject was implanted in all studies as a stand-alone procedure. In the study of Klamann et $\mathrm{al}^{41}$ the results were reported separately for patients with POAG and PEX. Numbers on the horizontal axis indicate the month of visit. Abbreviations: IOP, intraocular pressure; POAG, primary open-angle glaucoma; PEX, exfoliative glaucoma; BM, medicated baseline IOP; BU, unmedicated baseline IOP; $U$, unmedicated; $M$, medicated. 


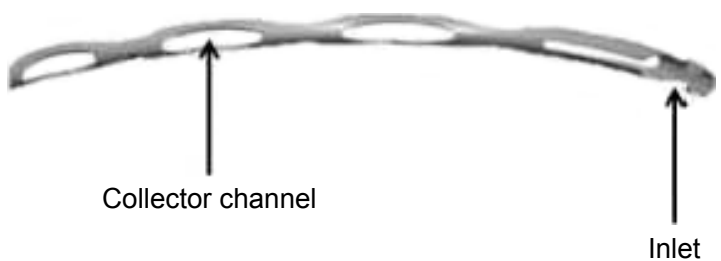

Figure 5 The Hydrus ${ }^{\circledR}$ micro-stent.

being inserted on a small guidewire with a special tip that separates the iris from the scleral spur (Figure 6). Openings along the whole length of the micro-stent allow aqueous humor outflow into the suprachoroidal space.

\section{Surgical procedure with the CyPass micro-stent} CyPass is implanted through a $1.5 \mathrm{~mm}$ clear corneal incision. Usually acetylcholine is injected into the anterior chamber to achieve a strong miosis. Then, a viscoelastic substance is injected in order to provide a stable anterior chamber. The procedure is controlled using a gonioscopy lens. The microdevice is inserted on a guidewire between the ciliary body and sclera until the suprachoroidal space is reached and the retention features are then released. The viscoelastic substance is removed via irritation and aspiration.

Three studies have been published on efficacy and safety of CyPass in patients with mild to moderate OAG. ${ }^{19,43,44}$ In a study published by Hoeh et al CyPass was implanted in a combined procedure with cataract extraction and the implantation of an IOL. ${ }^{19}$ In another study published by García-Feijoo et al CyPass was implanted in patients with phakic and pseudophakic eyes. ${ }^{43}$ In both studies the procedure was performed without a wash-out period of the medication used. In a third study published most recently by Vold et al the supraciliary micro-stent (CyPass) was implanted in a combined procedure with a cataract extraction and phacoemulsification. ${ }^{44}$

\section{Efficacy of CyPass}

The first study involved 167 eyes of 142 patients. Mean baseline IOP of the patient cohort was $20.2 \pm 6.0 \mathrm{mmHg} .{ }^{19}$ The eyes were analyzed in two subgroups - one with

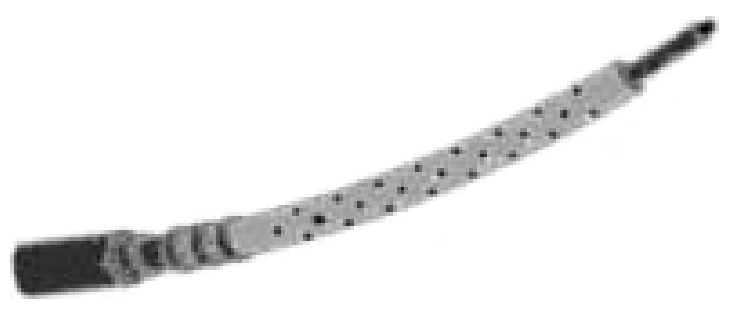

Figure 6 The CyPass ${ }^{\circledR}$ micro-stent placed on a guidewire. medicated baseline IOP levels of $\geq 21 \mathrm{mmHg}$ and another with baseline IOP levels of $<21 \mathrm{mmHg}$. Overall, mean IOP decreased from $20.2 \pm 6.0 \mathrm{mmHg}$ to $15.9 \pm 3.1 \mathrm{mmHg}$ at 12 months. Mean IOP decreased in the subgroup of patients with higher baseline IOP levels from $25.9 \pm 5.4 \mathrm{mmHg}$ to $16.3 \pm 3.4 \mathrm{mmHg}$, and in the subgroup of patients with lower IOP baseline levels from $16.6 \pm 2.7 \mathrm{mmHg}$ to $15.7 \pm 3.0 \mathrm{mmHg}$ (Figure 7). The number of medications used dropped from 2.1 at baseline to 1.1 at month 12 in the patient subgroup with higher baseline IOPs and from 2.0 at baseline to approximately 0.4 at month 12 in patients with lower IOPs. The second study involved 65 eyes from which 55 eyes were analyzed at the 12-month visit. ${ }^{43}$ This study was performed as a stand-alone procedure in patients with phakic or pseudophakic eyes. After 12 months, mean IOP was decreased from $24.5 \pm 2.8 \mathrm{mmHg}$ to $16.4 \pm 5.5 \mathrm{mmHg}$ (Figure 7) while the number of medications was reduced from 2.2 on average at baseline to 1.4 at month 12 . The third study was a multicenter, interventional randomized trial. ${ }^{44}$ In this study, subjects with $\mathrm{OAG}$ and cataract with mean diurnal IOP between $21 \mathrm{mmHg}$ and $33 \mathrm{mmHg}$ were randomized to phacoemulsification only $(n=131)$ or supraciliary stenting phacoemulsification and the implantation of a CyPass stent to the supraciliary space $(n=374)$. Mean preoperative IOP in the phacoemulsification and the micro-stent arm were $24.5 \pm 3.0 \mathrm{mmHg}$ and $24.4 \pm 2.8 \mathrm{mmHg}$, respectively. The mean numbers of medications in the two arms at baseline were comparable with $1.3 \pm 1.0$ and $1.4 \pm 0.9$ medications in the phacoemulsification and the microstent arm, respectively. At month 24, IOP was reduced by $7.4 \mathrm{mmHg}$ in the micro-stent arm versus $5.4 \mathrm{mmHg}$ in the phacoemulsification arm. Of the patients in the micro-stent arm, $85 \%$ did not require medications at month 24 and the mean number of medications was reduced by $67 \%(0.2 \pm 0.6$ medications). Seventy-seven percent of subjects in the micro-stent arm and $60 \%$ of subjects in the phacoemulsification arm achieved an unmedicated IOP decrease versus baseline of $\geq 20 \%$.

\section{Safety profile and adverse events of CyPass}

In the study by Hoeh et al the two most frequent complications were early hypotony in $13.8 \%$ of patients, which resolved within 1 month and an IOP increase of $\geq 10.0 \mathrm{mmHg}$ $(3.0 \%) .{ }^{19}$ An obstruction of the implant was seen in 5.4\% of the patients. García-Feijoo et al reported $11 \%$ of cases with IOP levels of $>30 \mathrm{mmHg}$ which persisted more than 1 month after the procedure. Cataract progression (12.2\%) and transient hyphema (6.2\%) were other adverse events reported in this study. ${ }^{43}$ In the study of Vold et al $37 \%$ of subjects in the micro-stent arm and $36 \%$ of the phacoemulsification arm experienced ocular adverse events through 


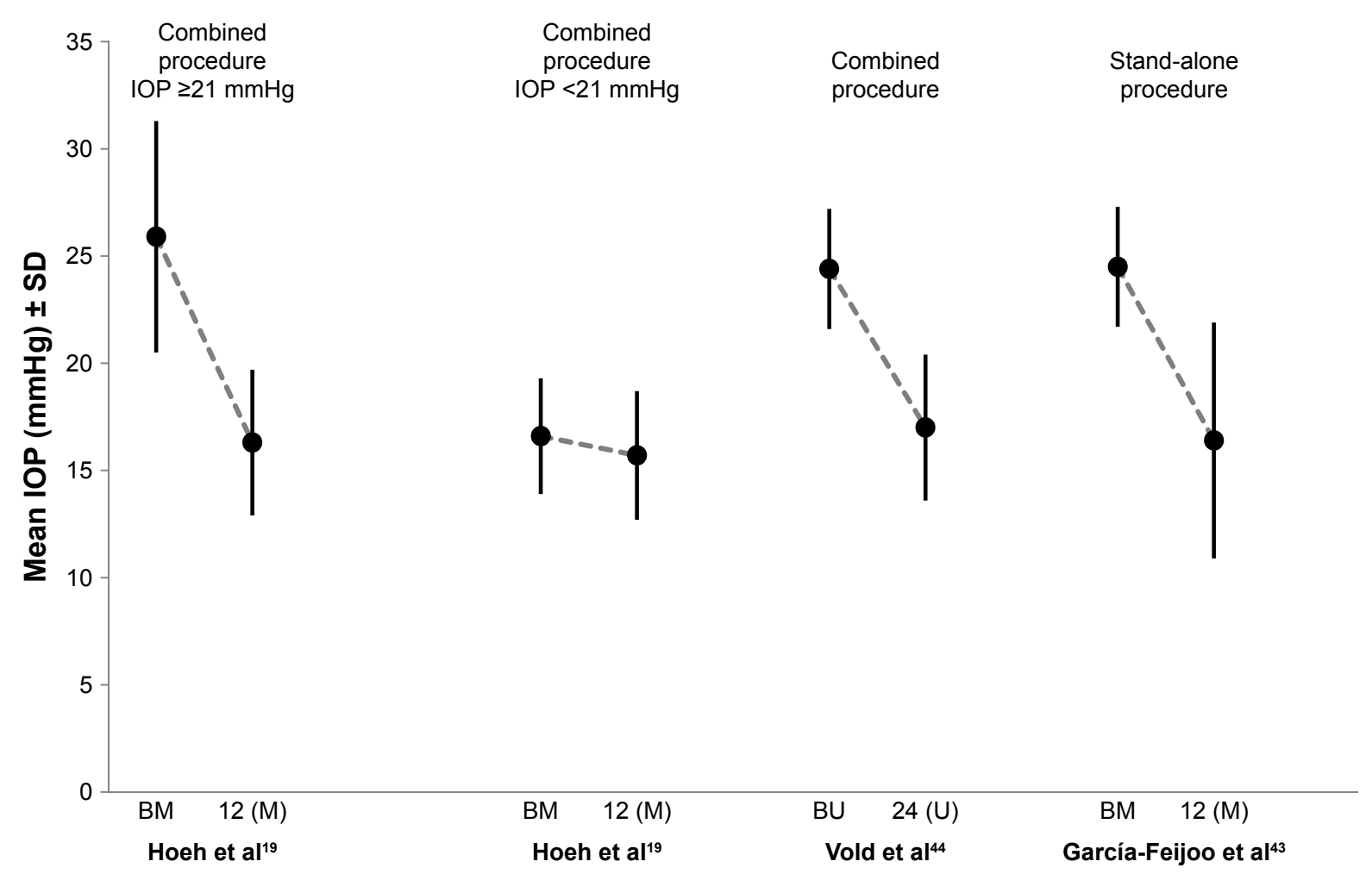

Figure 7 Mean IOP \pm SD at baseline and 12 months after the implantation of CyPass.

Notes: In the studies of Hoeh et $\mathrm{a}^{19}$ and Vold et $\mathrm{a}^{14}$ the implantation of CyPass was performed as a combined procedure during a cataract extraction with phacoemulsification and the implantation of an intraocular lens. In the study of García-Feijoo et al ${ }^{43}$ CyPass was implanted in a stand-alone procedure in phakic and pseudophakic eyes. Numbers on horizontal axis indicate the month of visit.

Abbreviations: IOP, intraocular pressure; BM, medicated baseline IOP; BU, unmedicated baseline IOP; M, medicated; U, unmedicated

month 24. Most frequently reported adverse events were transient ( $\leq 30$-day duration) best-corrected visual acuity loss of $\geq 2$ lines lasting up to 24 months ( $8.8 \%$ micro-stent arm, $15.3 \%$ phacoemulsification arm), iritis (8.6\% micro-stent arm, 3.8\% phacoemulsification arm), and corneal edema (3.5\% micro-stent arm, $1.5 \%$ phacoemulsification arm). Of micro-stent-related adverse events, eight stent obstructions $(2.1 \%)$, two instances of malpositioning, and two instances of migration/dislocation were observed.

\section{MIGS procedure opening a subconjunctival filtration pathway}

Subconjunctival filtration generates a new, non-physiological pathway for the outflow of aqueous humor. This route for outflow increase is already the basis for conventional trabeculectomy and for glaucoma epibulbar shunt surgeries.

\section{XEN gel stent}

The XEN gel stent (Allergan plc, Dublin, Ireland) is made of porcine collagen cross-linked with glutaraldehyde. The stent is $6 \mathrm{~mm}$ in length and has a lumen diameter of $45 \mu \mathrm{m}$. The stent is stiff when dehydrated but becomes soft and flexible when it comes into contact with the aqueous humor. Originally three different lumen diameters of the stent were investigated ( $45 \mu \mathrm{m}, 63 \mu \mathrm{m}$, and $140 \mu \mathrm{m})$, however, only the $45 \mu \mathrm{m}$ type is currently being further examined in ongoing clinical trials (Table 1). The outflow volume follows Poiseuille's law of laminar flow, where the diameter and length of the tube define the amount of outflow. The procedure is limited to eyes without conjunctival scarring.

\section{Surgical procedure with the XEN gel stent}

The implantation procedure can be done as stand-alone, or in combination with cataract surgery. The XEN gel stent is placed through a small, self-sealing, clear corneal incision using an inserter. The device is implanted into the subconjunctival space opposite the incision, thus, the procedure does not disrupt the conjunctival and subconjunctival tissue. A fistula is created, resulting in a bleb. Because this bleb is a significant risk factor for scar formation, the use of an antimetabolite such as mitomycin C (MMC) is recommended. Thus, MMC $10 \mu \mathrm{g}$ is usually injected under the conjunctiva approximately 20 minutes before the procedure.

Only one clinical study with the $45 \mu \mathrm{m}$ XEN gel stent has been published recently. Effectiveness and safety of phacoemulsification combined with the XEN implantation 
surgery was evaluated in patients with cataract and OAG in a prospective, 12-month follow-up study involving 30 eyes with at least two medications to control IOP. The combined procedure was performed through two temporal incisions after administering subconjunctival mitomycin. ${ }^{45}$

\section{Efficacy of XEN gel stent}

The mean preoperative IOP was $21.2 \pm 3.4 \mathrm{mmHg}$, with an average of 3.1 drugs. At month 12, IOP decreased by $29.3 \%$ to $15.0 \mathrm{mmHg}$, which is equivalent to a $6.2 \mathrm{mmHg}$ IOP decrease. Unfortunately there was no parallel group in which the effect of phacoemulsification and cataract surgery could be evaluated.

\section{Safety profile and adverse events of XEN gel stent} Overall, adverse events occurred in three eyes. Two eyes did not complete the procedure $\left(280^{\circ}\right.$ subconjunctival hemorrhage and XEN extrusion during repositioning). The third case had an encapsulation of the bleb 5 months after the surgery. Currently there is very limited further information about the safety profile of XEN gel stent. However, the implantation of the XEN gel stent can be regarded as a modified TE which results in a bleb, which requires the use of MMC, and thus it shares at least some of the risks of TE. Further long-term studies are needed to provide a full picture on the safety profile of XEN gel stent.

\section{Discussion}

Glaucoma therapy includes several different methods to lower IOP, such as medications, laser, and surgery. In many cases, patients need more than one of those treatments to achieve target-pressures low enough to avoid progression. Current management of glaucoma also comprises a variety of different issues. Major issues of medical therapy include treatment adherence and persistence issues, ${ }^{11,46}$ especially in patients treated concomitantly with different glaucoma medications, the toxicity of preservatives in eye-drops, such as benzalkonium chloride to corneal and conjunctival tissues, ${ }^{47}$ especially when applied chronically, and a poor tolerance of the applied glaucoma medications. ${ }^{8}$ MIGS may provide a solution for many of these issues. The different devices lower IOP between 3.2 and $12.6 \mathrm{mmHg}$. The reduction of IOP seems to be larger in patients with higher baseline IOP levels. ${ }^{19,37}$ Furthermore, the magnitude of the IOP lowering effect seems to depend on the number of shunts or stents which are implanted. ${ }^{35,36}$ However, further studies have to confirm these observations. Several limitations to the current findings are discussed in the various publications. These include limited data quality, especially in some studies with retrospective nature of the data, lack of study standardization, concomitant application of different therapies (ie, PE/IOL and micro-stents), limited knowledge on the duration of the IOP lowering effect for some microdevices, and missing information about the ideal patient for the different MIGS procedures. Concomitant application of different therapies in clinical studies with glaucoma devices frequently makes it difficult to do a proper evaluation and comparison of the results obtained. Direct comparisons of the evidence of the different approaches of MIGS are difficult or even impossible due to the diverse study designs, patient populations, and outcome measures. Thus a standardization of those studies is urgently needed. Key among the recommendations of the American National Standards Institute for those studies, is to apply a medication wash-out both at enrollment and at the follow-up visits, to mask the study for the tonometer reading and to provide a follow-up of at least 2 years. ${ }^{48}$ Many trials have included cataract surgery, therefore it is important when assessing the available MIGS data to consider the effect of PE/IOL itself on the decrease of IOP and on the number of IOP lowering medications needed after a combined PE/IOL/MIGS procedure. ${ }^{49-51}$ In the Ocular Hypertension Treatment Study, an initial IOP reduction was observed within the first 12 months. Despite the fact that this IOP lowering effect diminished over time, it remained present for up to 3 years. ${ }^{52}$ Therefore, clinicians cannot assume that IOP lowering abilities will be similar when micro-shunts are used during stand-alone procedures. In December 2015 the FDA issued guidance which specifies standards which should be met by future MIGS studies. ${ }^{53}$ These standards include a clear selection of patients with mild to moderate glaucoma by applying specific visual field and optic nerve characteristics, a follow-up period of at least 12 months, selection of glaucoma patients which allows a wash-out period at baseline and follow-up visits, diurnal IOP measurements, and a primary effectiveness endpoint of a $\geq 20 \%$ IOP reduction versus baseline. If future MIGS studies follow these proposed, more standardized study designs, a better understanding of the IOP reducing effects of each MIGS procedure will be possible.

When considering choosing the right patient profile for the different MIGS procedures, a few things besides the IOP lowering effect need be considered: firstly, the mode of action may be one criterion. iStent, iStent inject, and Hydrus work by improving aqueous humor outflow at the structure of the physiological outflow into Schlemm's canal, while the other options are generating new and thus probably less physiological outflow pathways into the suprachoroidal space (CyPass and iStent Supra [Glaukos Inc.]) or the 
Table 4 Implants used during micro-invasive glaucoma surgery procedures - summary of mode of action, possible risks, and potential use

\begin{tabular}{|c|c|c|c|c|c|}
\hline \multirow{2}{*}{$\begin{array}{l}\text { Outflow pathway } \\
\text { Devices }\end{array}$} & \multicolumn{3}{|c|}{ Trabecular } & \multirow{2}{*}{$\begin{array}{l}\text { Suprachoroidal } \\
\text { CyPass }^{\circledR}\end{array}$} & \multirow{2}{*}{$\begin{array}{l}\text { Subconjunctival } \\
\text { XEN }\end{array}$} \\
\hline & iStent $^{\circledR}$ & iStent $^{\circledR}$ inject & Hydrus & & \\
\hline Mode of action & \multicolumn{3}{|c|}{$\begin{array}{l}\text { Improvement of aqueous humor outflow } \\
\text { into Schlemm's canal }\end{array}$} & $\begin{array}{l}\text { Creation of an artificial outflow } \\
\text { for the aqueous humor into the } \\
\text { suprachoroidal space }\end{array}$ & $\begin{array}{l}\text { Formation of an artificial aqueous } \\
\text { humor outflow path to the } \\
\text { subconjunctival space }\end{array}$ \\
\hline Possible risks & \multicolumn{3}{|c|}{$\begin{array}{l}\text { - Lower risk for hypotony } \\
\text { - Peripheral anterior synechiae (Hydrus) }\end{array}$} & $\begin{array}{l}\text { - Higher risk for (transient) } \\
\text { hypotony } \\
\text { - (Transient) hyphema }\end{array}$ & $\begin{array}{l}\text { - Higher risk for (transient) hypotony } \\
\text { - Bleb formation and use of MMC } \\
\text { - Subconjunctival hemorrhage }\end{array}$ \\
\hline Potential use & \multicolumn{3}{|c|}{$\begin{array}{l}\text { - Mild to moderate glaucoma } \\
\text { - Patients with lower baseline IOP levels } \\
\text { - Patients with multiple glaucoma } \\
\text { medications, intolerance to glaucoma } \\
\text { medications, and/or adherence issues }\end{array}$} & $\begin{array}{l}\text { - Mild to moderate glaucoma } \\
\text { - Patients with higher baseline IOP } \\
\text { levels }\end{array}$ & $\begin{array}{l}\text { - More severe/advanced glaucoma } \\
\text { - Patients with higher baseline IOP } \\
\text { levels } \\
\text { - Need for lower target IOP levels } \\
\text { - Patients in whom target IOP is not } \\
\text { achieved by trabecular stents }\end{array}$ \\
\hline
\end{tabular}

Notes: iStent, iStent inject - Glaukos Inc., Laguna Hills, CA, USA; Hydrus - Ivantis Inc., Irvine, CA, USA; CyPass - Alcon Inc., Fort Worth, TX, USA; XEN - Allergan plc, Dublin, Ireland.

Abbreviations: IOP, intraocular pressure; MMC, mitomycin C.

subconjunctival space (XEN). Secondly, the safety profile of the different approaches needs to be considered, especially the risk for generating hypotony. Furthermore, the implantation of XEN gel stent can be regarded as a modified ab interno TE with the formation of a bleb and the need for MMC. A result of these considerations may be to use the Schlemm's canal micro-stents in patients with mild to moderate glaucoma, and the suprachoroidal and subconjunctival devices for the more severe cases of glaucoma (Table 4).

Schlemm's canal is a special feature. Within the scope of the concept of a segmental flow, the trabecular bypass operations may not achieve very low IOP levels as compared to TE. This is probably due to the fact that in patients with POAG, the number of opened collector channels remains the same with increased IOP and therefore may be the cause of an increased outflow resistance. ${ }^{54,55}$ Suprachoroidal devices may also be considered if trabecular stents fail or if the target pressure cannot be achieved by these stents. Further standardized studies are needed to gain more specific information on which patients will benefit most from each of these micro-devices. These data will help to individualize the management of glaucoma patients and to choose the best option MIGS for the individual glaucoma patient. Further information is also needed to choose the best medical approach with an appropriate primary mode of action (decrease aqueous humor production, increase of trabecular or uveoscleral outflow) in cases where adjunctive medication is needed.

\section{Acknowledgment}

Medical writing of this review was financially supported by Glaukos Inc., San Clemente, USA.

\section{Disclosure}

Medical writing of this review was done by eyecons (F Kimmich) with financial support from Glaukos Inc. San Clemente, USA. $\mathrm{C}$ Erb and A Jünemann are speakers for Glaukos Inc. L Pillunat has no conflicts of interest in this work.

\section{References}

1. Quigley HA, Broman AT. The number of people with glaucoma worldwide in 2010 and 2020. Br J Ophthalmol. 2006;90(3):262-267.

2. Chauhan BC, Mikelberg FS, Balaszi AG, LeBlanc RP, Lesk MR, Trope GE; Canadian Glaucoma Study Group. Canadian Glaucoma Study: 2. risk factors for the progression of open-angle glaucoma. Arch Ophthalmol. 2008;126(8):1030-1036.

3. Actis AG, Versino E, Brogliatti B, Rolle T. Risk factors for primary open angle glaucoma (POAG) progression: a study ruled in Torino. Open Ophthalmol J. 2016;10:129-139.

4. No authors listed. The Advanced Glaucoma Intervention Study (AGIS): 7. The relationship between control of intraocular pressure and visual field deterioration. The AGIS Investigators. Am J Ophthalmol. 2000;130(4):429-440.

5. Heijl A, Leske MC, Bengtsson B, Hyman L, Bengtsson B, Hussein M; Early Manifest Glaucoma Trial Group. Reduction of intraocular pressure and glaucoma progression: results from the Early Manifest Glaucoma Trial. Arch Ophthalmol. 2002;120(10):1268-1279.

6. European Glaucoma Society. Terminology and Guidelines for Glaucoma. 4th ed. Editrice Publicomm s.r.1; 2014.

7. Erb C, Gast U, Schremmer D. German register for glaucoma patients with dry eye. I. Basic outcome with respect to dry eye. Graefes Arch Clin Exp Ophthalmol. 2008;246(11):1593-1601.

8. Jaenen N, Baudouin C, Pouliquen P, Manni G, Figueiredo A, Zeyen T. Ocular symptoms and signs with preserved and preservative-free glaucoma medications. Eur J Ophthalmol. 2007;17(3):341-349.

9. Kass MA, Heuer DK, Higginbotham EJ, et al The Ocular Hypertension Treatment Study: a randomized trial determines that topical ocular hypotensive medication delays or prevents the onset of primary openangle glaucoma. Arch Ophthalmol. 2002;120(6):701-713.

10. Tsai JC, McClure CA, Ramos SE, Schlundt DG, Pichert JW. Compliance barriers in glaucoma: a systematic classification. J Glaucoma. 2003; 12(5):393-398. 
11. Tsai JC. A comprehensive perspective on patient adherence to topical glaucoma therapy. Ophthalmology. 2009;116(11 Suppl): S30-S36.

12. Friedman DS, Quigley HA, Gelb L, et al Using pharmacy claims data to study adherence to glaucoma medications: methodology and findings of the glaucoma adherence and persistency study (GAPS). Invest Ophthalmol Vis Sci. 2007;48(11):5052-5057.

13. Nordstrom BL, Friedman DS, Mozaffari E, Quigley HA, Walker AM. Persistence and adherence with topical glaucoma therapy. Am J Ophthalmol. 2005;140(4):598-606.

14. Saheb H, Ahmed II. Micro-invasive glaucoma surgery: current perspectives and future directions. Curr Opin Ophthalmol. 2012;23(2): 96-104.

15. Caprioli J, Kim JH, Friedman DS, et al Special commentary: supporting innovation for safe and effective minimally invasive glaucoma surgery: Summary of a Joint Meeting of the American Glaucoma Society and the Food and Drug Administration, Washington, DC, February 26, 2014. Ophthalmology. 2015;122(9):1795-1801.

16. Wellik SR, Dale EA. A review of the iStent $\left({ }^{\circledR}\right)$ trabecular micro-bypass stent: safety and efficacy. Clin Ophthalmol. 2015;9:677-684

17. Voskanyan L, García-Feijoó J, Belda JI, Fea A, Jünemann A, Baudouin C; Synergy Study Group. Prospective, unmasked evaluation of the iStent ${ }^{\mathbb{1}}$ inject system for open-angle glaucoma: synergy trial. $A d v$ Ther. 2014;31(2):189-201.

18. Pfeiffer N, Garcia-Feijoo J, Martinez-de-la-Casa JM, et al A randomized trial of a Schlemm's canal microstent with phacoemulsification for reducing intraocular pressure in open-angle glaucoma. Ophthalmology. 2015;122(7):1283-1293.

19. Hoeh H, Vold SD, Ahmed IK, et al Initial clinical experience with the CyPass micro-stent: safety and surgical outcomes of a novel supraciliary microstent. J Glaucoma. 2016;25(1):106-112.

20. Lewis RA. Ab interno approach to the subconjunctival space using a collagen glaucoma stent. J Cataract Refract Surg. 2014;40(8):1301-1306.

21. Gedde SJ, Schiffman JC, Feuer WJ, Herndon LW, Brandt JD, Budenz DL; Tube Versus Trabeculectomy Study Group. Three-year follow-up of the tube versus trabeculectomy study. Am J Ophthalmol. 2009; 148(5):670-684.

22. Johnson M. 'What controls aqueous humour resistance?'. Exp Eye Res. 2006;82(4):545-557.

23. Saccà $\mathrm{SC}$, Gandolfi $\mathrm{S}$, Bagnis $\mathrm{A}$, et al. The outflow pathway: $\mathrm{A}$ tissue with morphological and functional unity. Cell Physiol. 2016;231(19): 1876-1893.

24. Zeimer RC, Gieser DK, Wilensky JT, Noth JM, Mori MM, Odunukwe EE. A practical venomanometer. Measurement of episcleral venous pressure and assessment of the normal range. Arch Ophthalmol. 1983; 101(9):1447-1449.

25. Toris CB, Yablonski ME, Wang YL, Camras CB. Humor dynamics in the aging human eye. Am J Ophthalmol. 1999;127(4):407-412.

26. Sultan M, Blondeau P. Episcleral venous pressure in younger and older subjects in the sitting and supine positions. J Glaucoma. 2003;12(4): 370-373.

27. Selbach JM, Posielek K, Steuhl KP, Kremmer S. Episcleral venous pressure in untreated primary open-angle and normal-tension glaucoma. Ophthalmologica. 2005;219(6):357-361.

28. Fea AM. Phacoemulsification versus phacoemulsification with microbypass stent implantation in primary open-angle glaucoma: randomized double-masked clinical trial. J Cataract Refract Surg. 2010; 36(3):407-412.

29. Samuelson TW, Katz LJ, Wells JM, Duh YJ, Giamporcaro JE; US iStent Study Group. Randomized evaluation of the trabecular micro-bypass stent with phacoemulsification in patients with glaucoma and cataract. Ophthalmology. 2011;118(3):459-467.

30. Craven ER, Katz JL, Wells JM, Giamporcaro JE; iStent Study Group. Cataract surgery with trabecular micro-bypass stent implantation in patients with mild-to-moderate open-angle glaucoma and cataract: twoyear follow-up. J Cataract Refract Surg. 2012;38(8):1339-1345.
31. Arriola-Villalobos P, Martínez-de-la-Casa JM, Díaz-Valle D, Fernández-Pérez C, García-Sánchez J, García-Feijoó J. Combined iStent trabecular micro-bypass stent implantation and phacoemulsification for coexistent open-angle glaucoma and cataract: a long-term study. $\mathrm{Br} \mathrm{J}$ Ophthalmol. 2012;96(5):645-649.

32. Neuhann TH. Trabecular micro-bypass stent implantation during small-incision cataract surgery for open-angle glaucoma or ocular hypertension: Long-term results. J Cataract Refract Surg. 2015;41(12): 2664-2671.

33. Patel I, de Klerk TA, Au L. Manchester iStent study: early results from a prospective UK case series. Clin Exp Ophthalmol. 2013;41(7): $648-652$.

34. Fernández-Barrientos Y, Garcia-Feioó J, Martinez-de-la-Casa JM, Pablo LE, Fernández-Pérez C, García Sánchez J. Fluorophotometric study of the effect of the glaukos trabecular microbypass stent on aqueous humor dynamics. Invest Ophthalmol Vis Sci. 2010;51(7):3327-3332.

35. Belovay GW, Nagi A, Chan BJ, Rateb M, Ahmed II. Using multiple trabecular micro-bypass stents in cataract patients to treat open-angle glaucoma. J Cataract Refract Surg. 2012;38(11):1911-1917.

36. Katz LJ, Erb C, Carceller A, et al. Prospective, randomized study of one, two, or three trabecular bypass stents in open-angle glaucoma subjects on topical hypotensive medication. Clin Ophthalmol. 2015;9:2313-2320.

37. Ahmed II, Katz LJ, Chang DF, et al. Prospective evaluation of microinvasive glaucoma surgery with trabecular microbypass stents and prostaglandin in open-angle glaucoma. J Cataract Refract Surg. 2014;40(8): $1295-1300$.

38. Donnenfeld ED, Solomon KD, Voskanyan L, et al. A prospective 3-year follow-up trial of implantation of two trabecular microbypass stents in open-angle glaucoma. Clin Ophthalmol. 2015;9:2057-2065.

39. Ferguson TJ, Berdahl JP, Schweitzer JA, Sudhagoni RG. Clinical evaluation of a trabecular microbypass stent with phacoemulsification in patients with open-angle glaucoma and cataract. Clin Ophthalmol. 2016;10:1767-1773.

40. Seibold LK, Gamett KM, Kennedy JB, et al Outcomes after combined phacoemulsification and trabecular microbypass stent implantation in controlled open-angle glaucoma. J Cataract Refract Surg. 2016; 42(9): 1332-1338.

41. Klamann M, Gonnermann J, Pahlitzsch M, et al. iStent inject in phakic open angle glaucoma. Graefes Arch Clin Exp Ophthalmol. 2015;253(6): 941-947.

42. Fea AM, Belda JI, Rekas M, et al Prospective unmasked randomized evaluation of the iStent inject ${ }^{\circledR}$ versus two ocular hypotensive agents in patients with primary open-angle glaucoma. Clin Ophthalmol. 2014; $8: 875-882$.

43. García-Feijoo J, Rau M, Grisanti S, et al Supraciliary micro-stent implantation for open-angle glaucoma failing topical therapy: 1-year results of a multicenter study. Am J Ophthalmol. 2015;159(6):1075-1081.

44. Vold S, Ahmed II, Craven ER, et al Two-year COMPASS trial results: Supraciliary microstenting with phacoemulsification in patients with open-angle glaucoma and cataracts. Ophthalmology. 2016;123(10): 2103-2112.

45. Pérez-Torregrosa VT, Olate-Pérez Á, Cerdà-Ibáñez M, et al. Combined phacoemulsification and XEN45 surgery from a temporal approach and 2 incisions. Arch Soc Esp Oftalmol. 2016;91(9):415-421.

46. Lafuma A, Salmon JF, Robert J, Berdeaux G. Treatment persistence and cost-effectiveness of latanoprost/latanoprost-timolol, bimatoprost/ bimatoprost-timolol and travoprost/travoprost-timolol in glaucoma: an analysis based on the United Kingdom general practitioner research database. Clin Ophtlmol. 2011;5:361-367.

47. Baudouin C, Labbé A, Liang H, Pauly A, Brignole-Baudouin F. Preservatives in eyedrops: the good, the bad and the ugly. Prog Retin Eye Res. 2010;29(4):312-334.

48. American National Standards Institute. ANSI Z80. 27-2001 (R2014). Ophthalmics: Aqueous shunts for Glaucoma Application. Alexandria, VA: American National Standards Institute; 2001. 
49. Shingleton BJ, Gamell LS, O’Donoghue MW, Baylus SL, King R. Long-term changes in intraocular pressure after clear corneal phacoemulsification: normal patients versus glaucoma suspects and glaucoma patients. J Cataract Refract Surg. 1999;25(7):885-890.

50. Hayashi K, Hayashi H, Nakao F, Hayashi F. Effect of cataract surgery on intraocular pressure control in glaucoma patients. $J$ Cataract Refract Surg. 2001;27(11):1779-1786.

51. Poley BJ, Lindstrom RL, Samuelson TW. Long-term effects of phacoemulsification with intraocular lens implantation in normotensive and ocular hypertensive eyes. J Cataract Refract Surg. 2008;34(5): $735-742$.

52. Manberger SL, Gordon MO, Jampel H, et al Reduction in intraocular pressure after cataract extraction: the Ocular Hypertension Treatment Study. Ophthalmology. 2012;119(9):1826-1831.
53. Food and Drug Administration. Premarket Studies of Implantable Minimally Invasive Glaucoma Surgical (MIGS) Devices: Draft Guidance for Industry and Food and Drug Administration Staff. Rockville: Food and Drug Administration; 2015. Available from: http://www. fda.gov/downloads/MedicalDevices/DeviceRegulationandGuidance/ GuidanceDocuments/UCM433165.pdf. Accessed: May 11, 2017.

54. Hann CR, Vercnocke AJ, Bentley MD, Jorgensen SM, Fautsch MP. Anatomic changes in Schlemm's canal and collector channels in normal and primary open-angle glaucoma eyes using low and high perfusion pressures. Invest Ophthalmol Vis Sci. 2014;55(9):5834-5841.

55. Swaminathan SS, Oh DJ, Kang MH, Rhee DJ. Aqueous outflow: segmental and distal flow. J Cataract Refract Surg. 2014;40(8):1263-1272.
Clinical Ophthalmology

\section{Publish your work in this journal}

Clinical Ophthalmology is an international, peer-reviewed journal covering all subspecialties within ophthalmology. Key topics include: Optometry; Visual science; Pharmacology and drug therapy in eye diseases; Basic Sciences; Primary and Secondary eye care; Patient Safety and Quality of Care Improvements. This journal is indexed on

Submit your manuscript here: http://www.dovepress.com/clinical-ophthalmology-journal

\section{Dovepress}

PubMed Central and CAS, and is the official journal of The Society of Clinical Ophthalmology (SCO). The manuscript management system is completely online and includes a very quick and fair peer-review system, which is all easy to use. Visit http://www.dovepress.com/ testimonials.php to read real quotes from published authors. 